\title{
ON THE LOCAL EXTENSION OF KILLING VECTOR-FIELDS IN RICCI FLAT MANIFOLDS
}

\author{
ALEXANDRU D. IONESCU AND SERGIU KLAINERMAN
}

\section{Contents}

1. Introduction

2. Proof of Theorem 1.2

2.1. Tensorial equations

2.2. Carleman inequalities and the local extension theorem

3. Proof of Theorem 1.3

3.1. Explicit calculations

3.2. The metric $\widetilde{h}$

3.3. The space-time metric

4. Extension across null hypersurfaces

4.1. An extendibility criterion

4.2. A non-extendible example

References

\section{INTRODUCTION}

In this paper we revisit the extension problem for Killing vector-fields in smooth Ricci flat Lorentzian manifolds and its relevance to the black hole rigidity problem. In the most general situation the problem can be stated as follows:

Assume (M,g) is a given smooth pseudo-riemannian manifold, $O \subseteq \mathbf{M}$ is an open subset, and $Z$ is a smooth Killing vector-field in $O$. Under what assumptions does $Z$ extend (uniquely) as a Killing vector-field in $\mathbf{M}$ ?

A classical result 11 of Nomizu establishes such a unique extension provided that the metric is real analytic, $\mathbf{M}$ and $O$ are connected and $\mathbf{M}$ is simply connected. The result has been used (see [5] and [4]) to reduce the black hole rigidity problem, for real analytic stationary solutions of the Einstein field equations, to the simpler case of axial symmetry treated by the Carter-Robinson theorem. This reduction has been often regarded as decisive, especially in the physics literature, without a clear understanding of the sweeping simplification power of the analyticity assumption. Indeed, the remarkable thing about Nomizu's theorem, to start with, is the fact

Received by the editors August 22, 2011 and, in revised form, August 18, 2012.

2010 Mathematics Subject Classification. Primary 53B30, 83C05, 83C57.

The first author was supported in part by a Packard fellowship.

The second author was supported in part by NSF grant 0601186 as well as by the Fondation des Sciences Mathématiques de Paris.

Both authors were also supported in part by NSF-FRG grant DMS-1065710.

${ }^{1}$ See [10]. We rely here on the version of the theorem given in [4]. 
that the metric is not assumed to satisfy any specific equation. Moreover, no assumptions are needed about the boundary of $O$ in $\mathbf{M}$ and the result is global with only minimal assumptions on the topology of $\mathbf{M}$ and $O$. All of these are clearly wrong in the case of smooth manifolds $(\mathbf{M}, \mathbf{g})$ which are not real analytic. To be able to say anything meaningful we need to both restrict the metric $\mathbf{g}$ by realistic equations and make specific assumptions about the boundary of $O$. Local and global assumptions also need to be carefully separated.

In this paper we limit our attention to a purely local description of the extension problem in the smooth case. Throughout the paper we assume that $(\mathbf{M}, \mathbf{g})$ is a non-degenerate Ricci flat, pseudo-riemannian metric, i.e.,

$$
\operatorname{Ric}(\mathbf{g})=0 .
$$

We recall the following crucial concept.

Definition 1.1. A domain $O \subset \mathbf{M}$ is said to be strongly pseudo-convex at a boundary point $p \in \partial O$ if it admits a strongly pseudo-convex defining function $f$ at $p$, in the sense that there is an open neighborhood $U$ of $p$ in $\mathbf{M}$ and a smooth function $f: U \rightarrow \mathbb{R}, \nabla f(p) \neq 0$, such that $O \cap U=\{x \in U: f(x)<0\}$ and

$$
\mathbf{D}^{2} f(X, X)(p)<0
$$

for any $X \neq 0 \in T_{p}(\mathbf{M})$ for which $X(f)(p)=0$ and $\mathbf{g}_{p}(X, X)=0$.

It is easy to see that this definition, in particular (1.2), does not depend on the choice of the defining function $f$. The strong pseudo-convexity condition is automatically satisfied if the metric $\mathbf{g}$ is Riemannian. It is also satisfied for Lorentzian metrics $\mathbf{g}$ if $\partial O$ is space-like at $p$, but it imposes serious restrictions for time-like hypersurfaces. It clearly fails if $\partial O$ is null in a neighborhood of $p$. Indeed in that case we can choose the defining function $f$ to be optical, i.e.,

$$
\mathbf{D}^{\alpha} f \mathbf{D}_{\alpha} f=0
$$

at all points of $\partial O$ in a neighborhood of $p$, and thus, choosing $X^{\alpha}=\mathbf{D}^{\alpha} f$, we have

$$
X^{\alpha} X^{\beta} \mathbf{D}_{\alpha} \mathbf{D}_{\beta} f=\frac{1}{2} X\left(\mathbf{D}^{\alpha} f \mathbf{D}_{\alpha} f\right)=0 .
$$

Besides a new extension result (see Theorem 1.2 below) this paper contains two local counterexamples. In our main such result (see Theorem 1.3) we show that at any point $p$ in the complement of the bifurcation sphere of the horizon of a Kerr spacetime $\mathcal{K}(m, a), 0<a<m$, with $\mathbf{T}, Z$ denoting the usual stationary and axially symmetric Killing vector-fields of $\mathcal{K}(m, a)$, one can find local extensions of the Kerr metric, which coincide with $\mathcal{K}(m, a)$ inside the black hole, and such that only $\mathbf{T}$ extends as a Killing vector-field to a full neighborhood of $p$. The condition $a>0$ is important in our proof, since our construction only works in the region where $\mathbf{T}$ is timelike, i.e., the ergo-region. It remains open whether or not a similar counterexample can be constructed for the Schwarzschild spacetimes $\mathcal{K}(m, 0)$.

We first state the following extension theorem:

Theorem 1.2. Assume that $(\mathbf{M}, \mathbf{g})$ is a smooth d-dimensional Ricci flat, pseudoriemannian manifold and $O \subseteq \mathbf{M}$ is a strongly pseudo-convex domain at a point $p \in \partial O$. We assume that the metric $\mathbf{g}$ admits a smooth Killing vector-field $Z$ in $O$. Then $Z$ extends as a Killing vector-field for $\mathbf{g}$ to a neighborhood of the point $p$ in $\mathbf{M}$. 
Under more restrictive assumptions, a similar result was proved in [1] as a key component of a theorem on the uniqueness of the Kerr solution in [2]. In this paper we present a different, more geometric proof, which is valid in all dimensions and for all pseudo-riemannian metrics. More importantly, the proof we present here does not require that the vector-field $Z$ be tangent to the boundary $\partial O$ in a neighborhood of $p$, or the existence of a geodesic vector-field $L$, defined in a neighborhood of $p$, and commuting with $Z$ in $O$.

In applications, one would like to use Theorem 1.2 repeatedly and extend the Killing vector-field $Z$ to larger and larger open sets. For this it is important to understand the "size" of the implied neighborhood in the conclusion of the theorem, where the vector-field $Z$ extends. The proof shows that this neighborhood depends only on smoothness parameters of $\mathbf{g}$ and $f$ in a neighborhood of $p$ (see (2.21)), and a quantitative form of strong pseudo-convexity described in Lemma 2.12. The neighborhood does not depend in any way on the vector-field $Z$ itself.

In view of Theorem 1.2, Killing vector-fields extend locally across strongly pseudo-convex hypersurfaces in Ricci flat manifolds. A natural question is whether the strong pseudo-convexity condition is needed. We give a partial answer in Theorem 4.3, in general one cannot expect to extend a Killing vector-field across a null hypersurface in a 4-dimensional Lorentz manifold 2

Our second main theorem provides a counterexample to extendibility, in the setting of the black hole rigidity problem. Let $(\mathcal{K}(m, a), \mathbf{g})$ denote the (maximally extended) Kerr space-time of mass $m$ and angular momentum $m a, 0 \leq$ $a<m$ (see [5] for definitions). Let $\mathbf{M}^{(\text {end })}$ denote an asymptotic region, $\mathbf{E}=$ $\mathcal{I}^{-}\left(\mathbf{M}^{(e n d)}\right) \cap \mathcal{I}^{+}\left(\mathbf{M}^{(e n d)}\right)$ the corresponding domain of outer communications, and $\mathcal{H}^{-}=\delta\left(\mathcal{I}^{+}\left(\mathbf{M}^{(e n d)}\right)\right.$ the boundary (event horizon) of the corresponding white hole 3 . Let $\mathbf{T}$ denote the stationary (timelike in $\left.\mathbf{M}^{(e n d)}\right)$ Killing vector-field of $(\mathcal{K}(m, a), \mathbf{g})$, and let $Z$ denote its rotational (with closed orbits) Killing vector-field.

Theorem 1.3. Assume that $0<a<m$ and $U_{0} \subseteq \mathcal{K}(m, a)$ is an open set such that

$$
U_{0} \cap \mathcal{H}^{-} \cap \overline{\mathbf{E}} \neq \emptyset \text {. }
$$

Then there is an open set $U \subseteq U_{0}$ diffeomorphic to the open unit ball $B_{1} \subseteq \mathbb{R}^{4}$, $U \cap \mathcal{H}^{-} \neq \emptyset$, and a smooth Lorentz metric $\widetilde{\mathbf{g}}$ in $U$ with the following properties:

$$
\widetilde{\mathbf{g}}_{\mathbf{R i c}}=0 \quad \text { in } U, \quad \mathcal{L}_{\mathbf{T}} \widetilde{\mathbf{g}}=0 \quad \text { in } U, \quad \widetilde{\mathbf{g}}=\mathbf{g} \quad \text { in } U \backslash \mathbf{E} ;
$$

(ii) the vector-field $Z$ does not extend to a Killing vector-field for $\widetilde{\mathbf{g}}$ in $U$.

In other words, one can modify the Kerr space-time smoothly, on one side of the horizon $\mathcal{H}^{-}$, in such a way that the resulting metric still satisfies the Einstein vacuum equations, has $\mathbf{T}$ as a Killing vector-field, but does not admit an extension of the Killing vector-field $Z$. This result illustrates one of the major difficulties one faces in trying to extend Hawking's rigidity result to the more realistic setting of smooth stationary solutions of the Einstein vacuum equations: unlike in the analytic situation, one cannot hope to construct an additional symmetry of stationary

\footnotetext{
${ }^{2}$ Such a hypersurface is not strongly pseudo-convex; see the discussion before Theorem 1.2

${ }^{3}$ A similar statement can be made on the future event horizon $\mathcal{H}^{+}$.
} 
solutions of the Einstein vacuum equations (as in Hawking's Rigidity Theorem) by relying only on the local information provided by the equations 4

We remark that the conclusion of Theorem 1.3 holds also when $a=m>0$, with the same proof.

The rest of the paper is organized as follows: in section 2 we prove Theorem 1.2 and in section 3 we prove Theorem 1.3 . In section 4 we consider extensions across null hypersurfaces in 4-dimensional Lorentz manifolds and prove two more theorems: Theorem 4.1 which provides a criterion for the extension of Killing vector-fields, and Theorem 4.3, which provides a general framework when extension is not possible.

\section{Proof of Theorem 1.2}

In [1] and [2] the extension of the Killing vector-field $Z$ was done according to the transport equation,

$$
[L, Z]=c_{0} L
$$

where $\mathbf{D}_{L} L=0$ and $c_{0}$ is constant. Consequently, we had to assume, in $O$, that $Z$ is not only Killing but that it also satisfies the additional assumption (2.1) with respect to a geodesic non-vanishing vector-field $L$. This could be arranged in the particular cases studied in [1] and [2, but imposes serious restrictions on $Z$ in the general case, particularly if $Z$ vanishes in a neighborhood of the point $p$. To avoid this restriction, in this paper we extend $Z$ according to the weaker condition

$$
\mathbf{D}_{L} \mathbf{D}_{L} Z=\mathbf{R}(L, Z) L,
$$

which would follow easily from (2.1), and is automatically satisfied if $Z$ is Killing.

More precisely, we construct first a smooth vector-field $L$ in a neighborhood of $p$ such that

$$
\mathbf{D}_{L} L=0, \quad L(f)(p)=1,
$$

and extend $Z$ to a neighborhood of $p$ by solving the second order differential system (2.2). Therefore, after restricting to a small neighborhood of $p$, we may assume that $Z, L$ are smooth vector-fields in $\mathbf{M}$ with the properties

$$
\mathbf{D}_{L} L=0 \quad \text { in } \mathbf{M}, \quad L^{\alpha} L^{\beta}\left(\mathbf{D}_{\alpha} \mathbf{D}_{\beta} Z_{\mu}-Z^{\rho} \mathbf{R}_{\rho \alpha \beta \mu}\right)=0 \text { in } \mathbf{M}, \quad \mathcal{L}_{Z} \mathbf{g}=0 \quad \text { in } O .
$$

It remains to prove that the deformation tensor $\pi=\mathcal{L}_{Z} \mathbf{g}$ vanishes in a neighborhood of $p$. We cannot do this, however, without establishing at the same time that the tensor $\mathcal{L}_{Z} \mathbf{R}$ also vanishes identically in $\mathbf{M}$. Our strategy is to derive a wave equation for $\mathcal{L}_{Z} \mathbf{R}$, or rather a suitable modification of it, coupled with a number of transport equations along the integral curves of $L$ for various tensorial quantities including $\pi$ itself. These equations will be used to prove that $\pi$ and $\mathcal{L}_{Z} \mathbf{R}$ have to vanish in a full neighborhood of $p$, provided that the strong pseudo-convexity assumption, which guarantees the unique continuation property, is satisfied.

\footnotetext{
${ }^{4}$ As mentioned earlier a local version of Hawking's Rigidity Theorem was proved in [1. The key additional information used in that paper is the existence of a regular bifurcation sphere, which is the smooth transversal intersection of two non-expanding horizons.
} 
2.1. Tensorial equations. We first consider the properties of $\mathcal{L}_{Z} \mathbf{R}$. Observe that $\mathcal{L}_{Z} \mathbf{R}$ verifies all the algebraic symmetries of $\mathbf{R}$ except the fact that, for an Einstein vacuum metric $\mathbf{g}, \mathbf{R}$ is traceless. We have instead,

$$
\mathrm{g}^{\alpha \gamma} \mathcal{L}_{Z} \mathbf{R}_{\alpha \beta \gamma \delta}=\pi^{\alpha \gamma} \mathbf{R}_{\alpha \beta \gamma \delta}
$$

To re-establish this property we can introduce (see also Chapter 7 in [3]) modifications 5 of $\mathcal{L}_{Z} \mathbf{R}$ of the form

$$
\widehat{\mathcal{L}}_{Z} \mathbf{R}:=\mathcal{L}_{Z} \mathbf{R}-B \odot \mathbf{R},
$$

where, for any given 2 -tensor $B$, we write

$$
(B \odot \mathbf{R})_{\alpha \beta \gamma \delta}:=B_{\alpha}{ }^{\lambda} \mathbf{R}_{\lambda \beta \gamma \delta}+B_{\beta}{ }^{\lambda} \mathbf{R}_{\alpha \lambda \gamma \delta}+B_{\gamma}{ }^{\lambda} \mathbf{R}_{\alpha \beta \lambda \delta}+B_{\delta}{ }^{\lambda} \mathbf{R}_{\alpha \beta \gamma \lambda} .
$$

It is easy to check that, for any 2 -tensor $B, B \odot \mathbf{R}$ verifies all the algebraic symmetries of the general Riemann curvature tensor, i.e.,

$$
\begin{aligned}
& (B \odot \mathbf{R})_{\alpha \beta \gamma \delta}=-(B \odot \mathbf{R})_{\beta \alpha \gamma \delta}=-(B \odot \mathbf{R})_{\alpha \beta \delta \gamma}=(B \odot \mathbf{R})_{\gamma \delta \alpha \beta}, \\
& (B \odot \mathbf{R})_{\alpha \beta \gamma \delta}+(B \odot \mathbf{R})_{\alpha \gamma \delta \beta}+(B \odot \mathbf{R})_{\alpha \delta \beta \gamma}=0 .
\end{aligned}
$$

Moreover, using the Einstein vacuum equations, we get

$$
\mathbf{g}^{\alpha \gamma}(B \odot \mathbf{R})_{\alpha \beta \gamma \delta}=B^{\mu \lambda}\left(\mathbf{R}_{\lambda \beta \mu \delta}+\mathbf{R}_{\mu \beta \lambda \delta}\right) .
$$

In particular, for any antisymmetric $B, B \odot \mathbf{R}$ is traceless, i.e., a Weyl field. We have proved the following:

Proposition 2.1. Assume $\omega$ is an antisymmetric 2-form in $\mathbf{M}$ and let

$$
W:=\mathcal{L}_{Z} \mathbf{R}-\frac{1}{2}(\pi+\omega) \odot \mathbf{R} .
$$

Then $W$ is a Weyl field in $\mathbf{M}$, i.e.,

$$
\begin{aligned}
& W_{\alpha \beta \gamma \delta}=-W_{\beta \alpha \gamma \delta}=-W_{\alpha \beta \delta \gamma}=W_{\gamma \delta \alpha \beta}, \\
& W_{\alpha \beta \gamma \delta}+W_{\alpha \gamma \delta \beta}+W_{\alpha \delta \beta \gamma}=0, \\
& \mathbf{g}^{\alpha \gamma} W_{\alpha \beta \gamma \delta}=0 .
\end{aligned}
$$

We shall next establish a divergence equation for $W$. We do this by commuting the divergence equation for $\mathbf{R}$ with $\mathcal{L}_{Z}$. We rely on the following (see Lemma 7.1.3 in [3]):

Lemma 2.2. For arbitrary $k$-covariant tensor-field $V$ and vector-field $X$ we have

$$
\mathbf{D}_{\beta}\left(\mathcal{L}_{X} V_{\alpha_{1} \ldots \alpha_{k}}\right)-\mathcal{L}_{X}\left(\mathbf{D}_{\beta} V_{\alpha_{1} \ldots \alpha_{k}}\right)=\sum_{j=1}^{k}{ }^{(X)} \Gamma_{\alpha_{j} \beta \rho} V_{\alpha_{1} \ldots}{ }^{\rho} \ldots \alpha_{k},
$$

where ${ }^{(X)} \pi=\mathcal{L}_{X} \mathbf{g}$ is the deformation tensor of $X$ and

$$
{ }^{(X)} \Gamma_{\alpha \beta \mu}=\frac{1}{2}\left(\mathbf{D}_{\alpha}{ }^{(X)} \pi_{\beta \mu}+\mathbf{D}_{\beta}{ }^{(X)} \pi_{\alpha \mu}-\mathbf{D}_{\mu}{ }^{(X)} \pi_{\alpha \beta}\right) .
$$

Definition 2.3. We denote $\pi={ }^{(Z)} \pi$ and $\Gamma={ }^{(Z)} \Gamma$ the corresponding tensors associated to the vector-field $Z$. We also introduce the tensors

$$
\begin{aligned}
& P_{\alpha \beta \mu}=(1 / 2)\left(\mathbf{D}_{\alpha} \pi_{\beta \mu}-\mathbf{D}_{\beta} \pi_{\alpha \mu}-\mathbf{D}_{\mu} \omega_{\alpha \beta}\right), \\
& B_{\alpha \beta}=(1 / 2)\left(\pi_{\alpha \beta}+\omega_{\alpha \beta}\right), \\
& W_{\alpha \beta \gamma \delta}=\left(\mathcal{L}_{Z} \mathbf{R}\right)_{\alpha \beta \gamma \delta}-(B \odot \mathbf{R})_{\alpha \beta \gamma \delta} .
\end{aligned}
$$

\footnotetext{
${ }^{5}$ Note, however, that, unlike 3], our $B$ here is not symmetric.
} 
All of these tensors depend on the 2-form $\omega$, which will be defined later (see (2.9)) to achieve a key cancellation in the proof of the transport equation (2.13) (see the identity $L^{\mu} P_{\alpha \beta \mu}=0$ in (2.10) $)$.

Using Lemma 2.2 we can now prove the following:

Lemma 2.4. The Weyl field $W$ verifies the divergence equation

$$
\begin{aligned}
\mathbf{D}^{\alpha} W_{\alpha \beta \gamma \delta}= & B^{\mu \nu} \mathbf{D}_{\nu} \mathbf{R}_{\mu \beta \gamma \delta}+\mathbf{g}^{\mu \nu} P_{\mu \rho \nu} \mathbf{R}^{\rho}{ }_{\beta \gamma \delta} \\
& +P_{\beta \nu \mu} \mathbf{R}^{\mu \nu}{ }_{\gamma \delta}+P_{\gamma \nu \mu} \mathbf{R}^{\mu}{ }_{\beta}{ }^{\nu}{ }_{\delta}+P_{\delta \nu \mu} \mathbf{R}^{\mu}{ }_{\beta \gamma}{ }^{\nu} .
\end{aligned}
$$

Proof of Lemma 2.4. Using Lemma 2.2 and the identity $\mathbf{D}^{\alpha} \mathbf{R}_{\alpha \beta \gamma \delta}=0$ (which is a consequence of the Einstein vacuum equations), we easily deduce

$$
\begin{aligned}
\mathbf{D}^{\alpha} \mathcal{L}_{Z} & \mathbf{R}_{\alpha \beta \gamma \delta}=\mathbf{g}^{\alpha \mu} \mathbf{D}_{\mu} \mathcal{L}_{Z} \mathbf{R}_{\alpha \beta \gamma \delta} \\
& =\mathbf{g}^{\alpha \mu}\left(\mathcal{L}_{Z} \mathbf{D}_{\mu} \mathbf{R}_{\alpha \beta \gamma \delta}+\Gamma_{\alpha \mu \rho} \mathbf{R}^{\rho}{ }_{\beta \gamma \delta}+\Gamma_{\beta \mu \rho} \mathbf{R}_{\alpha}{ }^{\rho}{ }_{\gamma \delta}+\Gamma_{\gamma \mu \rho} \mathbf{R}_{\alpha \beta}{ }^{\rho}{ }_{\delta}+\Gamma_{\delta \mu \rho} \mathbf{R}_{\alpha \beta \gamma}{ }^{\rho}\right) \\
& =\pi^{\alpha \mu} \mathbf{D}_{\mu} \mathbf{R}_{\alpha \beta \gamma \delta}+\Gamma^{\mu}{ }_{\mu \rho} \mathbf{R}^{\rho}{ }_{\beta \gamma \delta}+\Gamma_{\beta \mu \rho} \mathbf{R}^{\mu \rho}{ }_{\gamma \delta}+\Gamma_{\gamma \mu \rho} \mathbf{R}^{\mu}{ }_{\beta}{ }^{\rho}{ }_{\delta}+\Gamma_{\delta \mu \rho} \mathbf{R}^{\mu}{ }_{\beta \gamma}{ }^{\rho} .
\end{aligned}
$$

Using the definition and the Einstein vacuum equations, we derive

$$
\begin{aligned}
\mathbf{D}^{\alpha}(B \odot \mathbf{R})_{\alpha \beta \gamma \delta}= & B^{\alpha \lambda} \mathbf{D}_{\alpha} \mathbf{R}_{\lambda \beta \gamma \delta}+\mathbf{D}^{\alpha} B_{\alpha \lambda} \mathbf{R}^{\lambda}{ }_{\beta \gamma \delta}+\mathbf{D}_{\alpha} B_{\beta \lambda} \mathbf{R}^{\alpha \lambda}{ }_{\gamma \delta} \\
& +\mathbf{D}_{\alpha} B_{\gamma \lambda} \mathbf{R}^{\alpha}{ }_{\beta}{ }^{\lambda}{ }_{\delta}+\mathbf{D}_{\alpha} B_{\delta \lambda} \mathbf{R}^{\alpha}{ }_{\beta \gamma}{ }^{\lambda},
\end{aligned}
$$

for any 2-tensor $B$. Thus, if $B=(1 / 2)(\pi+\omega)$,

$$
\begin{aligned}
\mathbf{D}^{\alpha} W_{\alpha \beta \gamma \delta}= & \left(\pi^{\mu \nu}-B^{\mu \nu}\right) \mathbf{D}_{\mu} \mathbf{R}_{\nu \beta \gamma \delta}+\mathbf{g}^{\mu \nu}\left(\Gamma_{\mu \nu \rho}-\mathbf{D}_{\nu} B_{\mu \rho}\right) \mathbf{R}^{\rho}{ }_{\beta \gamma \delta} \\
& +\left(\Gamma_{\beta \mu \nu}-\mathbf{D}_{\mu} B_{\beta \nu}\right) \mathbf{R}^{\mu \nu}{ }_{\gamma \delta}+\left(\Gamma_{\gamma \mu \nu}-\mathbf{D}_{\mu} B_{\gamma \nu}\right) \mathbf{R}^{\mu}{ }_{\beta}{ }^{\nu}{ }_{\delta} \\
& +\left(\Gamma_{\delta \mu \nu}-\mathbf{D}_{\mu} B_{\delta \nu}\right) \mathbf{R}^{\mu}{ }_{\beta \gamma}{ }^{\nu} .
\end{aligned}
$$

We observe now that

$$
\Gamma_{b a c}-\mathbf{D}_{a} B_{b c}=P_{b c a},
$$

which completes the proof of the lemma.

We now look for transport equations for the tensor-fields $B, P$ appearing in (2.6), of the form

$$
\mathbf{D}_{L}(B, P)=\mathcal{M}(W, B, P),
$$

with the notation $\mathcal{M}(W, B, P)$ explained below.

Definition 2.5. By convention, we let $\mathcal{M}\left({ }^{(1)} B, \ldots,{ }^{(k)} B\right)$ denote any smooth "multiple" of the tensors ${ }^{(1)} B, \ldots,{ }^{(k)} B$, i.e., any tensor of the form

$$
\begin{aligned}
& \mathcal{M}\left({ }^{(1)} B, \ldots,{ }^{(k)} B\right)_{\alpha_{1} \ldots \alpha_{r}} \\
& \quad={ }^{(1)} B_{\beta_{1} \ldots \beta_{m_{1}}}{ }^{(1)} C_{\alpha_{1} \ldots \alpha_{r}}{ }^{\beta_{1} \ldots \beta_{m_{1}}}+\ldots+{ }^{(k)} B_{\beta_{1} \ldots \beta_{m_{k}}}{ }^{(k)} C_{\alpha_{1} \ldots \alpha_{r}}{ }^{\beta_{1} \ldots \beta_{m_{k}}},
\end{aligned}
$$

for some smooth tensors ${ }^{(1)} C, \ldots,{ }^{(k)} C$ in $\mathbf{M}$.

We start with a lemma.

Lemma 2.6. Given the vector-field $Z$, extended to $\mathbf{M}$ by (2.3), we have

$$
L^{\beta} \pi_{\alpha \beta}=0 \quad \text { in } \mathbf{M} \text {. }
$$

Moreover, if we define $\omega$ in $\mathbf{M}$ as the solution of the transport equation

$$
\mathbf{D}_{L} \omega_{\alpha \beta}=\pi_{\alpha \rho} \mathbf{D}_{\beta} L^{\rho}-\pi_{\beta \rho} \mathbf{D}_{\alpha} L^{\rho}
$$


with $\omega=0$ in $O$, then

$$
L^{\mu} P_{\alpha \beta \mu}=0, \quad L^{\beta} \omega_{\alpha \beta}=0 \quad \text { in } \quad \mathbf{M} .
$$

Proof of Lemma 2.6. We first remark that $L^{\alpha} L^{\beta} \mathbf{D}_{\alpha} Z_{\beta}=0$ in $\mathbf{M}$. Indeed, using (2.3), we get

$$
L^{\rho} \mathbf{D}_{\rho}\left(L^{\alpha} L^{\beta} \mathbf{D}_{\alpha} Z_{\beta}\right)=L^{\rho} L^{\alpha} L^{\beta} \mathbf{D}_{\rho} \mathbf{D}_{\alpha} Z_{\beta}=L^{\beta} L^{\alpha} L^{\rho} Z^{\nu} \mathbf{R}_{\nu \alpha \rho \beta}=0 .
$$

Since $L^{\alpha} L^{\beta} \mathbf{D}_{\alpha} Z_{\beta}=0$ in $O$ we deduce that

$$
L^{\alpha} L^{\beta} \mathbf{D}_{\alpha} Z_{\beta}=0 \quad \text { in } \mathbf{M} \text {. }
$$

We now prove (2.8). Using (2.3) and (2.11) we compute

$$
\begin{aligned}
L^{\rho} \mathbf{D}_{\rho}\left(L^{\beta} \pi_{\alpha \beta}\right) & =L^{\rho} L^{\beta}\left(\mathbf{D}_{\rho} \mathbf{D}_{\beta} Z_{\alpha}+\mathbf{D}_{\rho} \mathbf{D}_{\alpha} Z_{\beta}\right) \\
& =L^{\rho} L^{\beta} Z^{\mu} \mathbf{R}_{\mu \rho \beta \alpha}+L^{\rho} L^{\beta} \mathbf{D}_{\alpha} \mathbf{D}_{\rho} Z_{\beta}+L^{\rho} L^{\beta} Z^{\mu} \mathbf{R}_{\rho \alpha \beta \mu} \\
& =\mathbf{D}_{\alpha}\left(L^{\rho} L^{\beta} \mathbf{D}_{\rho} Z_{\beta}\right)-L^{\beta} \mathbf{D}_{\rho} Z_{\beta} \mathbf{D}_{\alpha} L^{\rho}-L^{\rho} \mathbf{D}_{\rho} Z_{\beta} \mathbf{D}_{\alpha} L^{\beta} \\
& =-L^{\beta} \pi_{\mu \beta} \mathbf{D}_{\alpha} L^{\mu} .
\end{aligned}
$$

Since $L^{\beta} \pi_{\alpha \beta}$ vanishes in $O$, it follows that $L^{\beta} \pi_{\alpha \beta}$ vanishes in $\mathbf{M}$, as desired.

The first identity in (2.10) follows from the definitions of $\omega$ and $P$ and the identity (2.8):

$$
\begin{aligned}
2 L^{\mu} P_{\alpha \beta \mu} & =L^{\mu} \mathbf{D}_{\alpha} \pi_{\beta \mu}-L^{\mu} \mathbf{D}_{\beta} \pi_{\alpha \mu}-L^{\mu} \mathbf{D}_{\mu} \omega_{\alpha \beta} \\
& =-\pi_{\beta \mu} \mathbf{D}_{\alpha} L^{\mu}+\pi_{\alpha \mu} \mathbf{D}_{\beta} L^{\mu}-L^{\mu} \mathbf{D}_{\mu} \omega_{\alpha \beta}=0 .
\end{aligned}
$$

To prove the second identity, we compute, using the definition (2.9) and the identities $L^{\beta} \pi_{\beta \rho}=0$ and $\mathbf{D}_{L} L=0$,

$$
\mathbf{D}_{L}\left(L^{\beta} \omega_{\alpha \beta}\right)=L^{\rho} L^{\beta} \mathbf{D}_{\rho} \omega_{\alpha \beta}=L^{\beta}\left(\pi_{\alpha \rho} \mathbf{D}_{\beta} L^{\rho}-\pi_{\beta \rho} \mathbf{D}_{\alpha} L^{\rho}\right)=0 .
$$

Since $L^{\beta} \omega_{\alpha \beta}$ vanishes in $O$, it follows that $L^{\beta} \omega_{\alpha \beta}$ vanishes in $\mathbf{M}$, as desired.

We derive now our main transport equations for the tensors $B$ and $P$.

Proposition 2.7. In $\mathbf{M}$ we have

$$
\mathbf{D}_{L} B_{\alpha \beta}=L^{\rho} P_{\rho \beta \alpha}-\mathbf{D}_{\alpha} L^{\rho} B_{\rho \beta}
$$

and

$$
\mathbf{D}_{L} P_{\alpha \beta \mu}=L^{\nu} W_{\alpha \beta \mu \nu}+L^{\nu} B_{\mu}{ }^{\rho} \mathbf{R}_{\alpha \beta \rho \nu}-\mathbf{D}_{\mu} L^{\rho} P_{\alpha \beta \rho} .
$$

Proof of Proposition 2.7. Using (2.8)-(2.10), we calculate

$$
\begin{aligned}
2 L^{\rho} \mathbf{D}_{\rho} B_{\alpha \beta} & =L^{\rho} \mathbf{D}_{\rho} \pi_{\beta \alpha}+L^{\rho} \mathbf{D}_{\rho} \omega_{\alpha \beta} \\
& =L^{\rho}\left(2 P_{\rho \beta \alpha}+\mathbf{D}_{\alpha} \omega_{\rho \beta}+\mathbf{D}_{\beta} \pi_{\rho \alpha}\right)+\pi_{\alpha \rho} \mathbf{D}_{\beta} L^{\rho}-\pi_{\beta \rho} \mathbf{D}_{\alpha} L^{\rho} \\
& =2 L^{\rho} P_{\rho \beta \alpha}-2 B_{\rho \beta} \mathbf{D}_{\alpha} L^{\rho},
\end{aligned}
$$

and the desired identity (2.12) follows.

To prove (2.13), we rely on the following identity:

$$
\begin{aligned}
\mathbf{D}_{\nu} \widetilde{P}_{\alpha \beta \mu}-\mathbf{D}_{\mu} \widetilde{P}_{\alpha \beta \nu} & =\left(\mathcal{L}_{Z} \mathbf{R}\right)_{\alpha \beta \mu \nu}-(1 / 2) \pi_{\alpha}{ }^{\rho} \mathbf{R}_{\rho \beta \mu \nu}-(1 / 2) \pi_{\beta}{ }^{\rho} \mathbf{R}_{\alpha \rho \mu \nu}, \\
\widetilde{P}_{\alpha \beta \mu} & :=(1 / 2)\left(\mathbf{D}_{\alpha} \pi_{\beta \mu}-\mathbf{D}_{\beta} \pi_{\alpha \mu}\right)=P_{\alpha \beta \mu}+(1 / 2) \mathbf{D}_{\mu} \omega_{\alpha \beta} .
\end{aligned}
$$


Assuming (2.14) we easily prove now (2.13). Indeed, relying on (2.14), (2.8), and the definitions we calculate

$$
\begin{aligned}
L^{\nu}\left(\mathbf{D}_{\nu} P_{\alpha \beta \mu}-\mathbf{D}_{\mu} P_{\alpha \beta \nu}\right) & =L^{\nu}\left[\frac{1}{2} \mathbf{D}_{\mu} \mathbf{D}_{\nu} \omega_{\alpha \beta}-\frac{1}{2} \mathbf{D}_{\nu} \mathbf{D}_{\mu} \omega_{\alpha \beta}+W_{\alpha \beta \mu \nu}+(B \cdot \mathbf{R})_{\alpha \beta \mu \nu}\right. \\
& \left.\quad-\frac{1}{2} \pi_{\alpha}{ }^{\rho} \mathbf{R}_{\rho \beta \mu \nu}-\frac{1}{2} \pi_{\beta}{ }^{\rho} \mathbf{R}_{\alpha \rho \mu \nu}\right] \\
& =L^{\nu} W_{\alpha \beta \mu \nu}+L^{\nu} B_{\mu}{ }^{\rho} \mathbf{R}_{\alpha \beta \rho \nu} .
\end{aligned}
$$

The identity (2.13) is now an immediate consequence of (2.10).

It remains to prove the identity (2.14). We have, in fact, the following more general version of it.

Lemma 2.8. Let $X$ be a vector-field with deformation tensor ${ }^{(X)} \pi$ and define

$$
{ }^{(X)} P=(1 / 2)\left(\mathbf{D}_{\alpha}{ }^{(X)} \pi_{\beta \mu}-\mathbf{D}_{\beta}{ }^{(X)} \pi_{\alpha \mu}\right) .
$$

Then,

$$
\begin{aligned}
\mathbf{D}_{\nu}{ }^{(X)} P_{\alpha \beta \mu}-\mathbf{D}_{\mu}{ }^{(X)} P_{\alpha \beta \nu}= & \left(\mathcal{L}_{X} \mathbf{R}\right)_{\alpha \beta \mu \nu}-(1 / 2)^{(X)} \pi_{\alpha}{ }^{\rho} \mathbf{R}_{\rho \beta \mu \nu} \\
& -(1 / 2)^{(X)} \pi_{\beta}{ }^{\rho} \mathbf{R}_{\alpha \rho \mu \nu} .
\end{aligned}
$$

Proof of Lemma 2.8. Using the definition of ${ }^{(X)} P$,

$$
\begin{aligned}
2 \mathbf{D}_{\nu}{ }^{(X)} P_{\alpha \beta \mu} & -2 \mathbf{D}_{\mu}{ }^{(X)} P_{\alpha \beta \nu} \\
& =\mathbf{D}_{\nu} \mathbf{D}_{\alpha}{ }^{(X)} \pi_{\beta \mu}-\mathbf{D}_{\nu} \mathbf{D}_{\beta}{ }^{(X)} \pi_{\alpha \mu}-\mathbf{D}_{\mu} \mathbf{D}_{\alpha}{ }^{(X)} \pi_{\beta \nu}+\mathbf{D}_{\mu} \mathbf{D}_{\beta}{ }^{(X)} \pi_{\alpha \nu} \\
& =\left(\mathbf{D}_{\nu} \mathbf{D}_{\alpha} \mathbf{D}_{\beta} X_{\mu}-\mathbf{D}_{\nu} \mathbf{D}_{\beta} \mathbf{D}_{\alpha} X_{\mu}\right)+\left(\mathbf{D}_{\nu} \mathbf{D}_{\alpha} \mathbf{D}_{\mu} X_{\beta}-\mathbf{D}_{\mu} \mathbf{D}_{\alpha} \mathbf{D}_{\nu} X_{\beta}\right) \\
& +\left(\mathbf{D}_{\mu} \mathbf{D}_{\beta} \mathbf{D}_{\alpha} X_{\nu}-\mathbf{D}_{\mu} \mathbf{D}_{\alpha} \mathbf{D}_{\beta} X_{\nu}\right)+\left(\mathbf{D}_{\mu} \mathbf{D}_{\beta} \mathbf{D}_{\nu} X_{\alpha}-\mathbf{D}_{\nu} \mathbf{D}_{\beta} \mathbf{D}_{\mu} X_{\alpha}\right) \\
& =I+I I+I I I+I V .
\end{aligned}
$$

We calculate

$$
I=X^{\rho} \mathbf{D}_{\nu} \mathbf{R}_{\alpha \beta \mu \rho}+\mathbf{R}_{\alpha \beta \mu \rho} \mathbf{D}_{\nu} X^{\rho}
$$

and

$$
\begin{aligned}
I I & =\left(\mathbf{D}_{\nu} \mathbf{D}_{\alpha} \mathbf{D}_{\mu} X_{\beta}-\mathbf{D}_{\alpha} \mathbf{D}_{\nu} \mathbf{D}_{\mu} X_{\beta}\right) \\
& +\left(\mathbf{D}_{\alpha} \mathbf{D}_{\nu} \mathbf{D}_{\mu} X_{\beta}-\mathbf{D}_{\alpha} \mathbf{D}_{\mu} \mathbf{D}_{\nu} X_{\beta}\right)+\left(\mathbf{D}_{\alpha} \mathbf{D}_{\mu} \mathbf{D}_{\nu} X_{\beta}-\mathbf{D}_{\mu} \mathbf{D}_{\alpha} \mathbf{D}_{\nu} X_{\beta}\right) \\
& =\left(\mathbf{R}_{\nu \alpha \mu \rho} \mathbf{D}^{\rho} X_{\beta}+\mathbf{R}_{\nu \alpha \beta \rho} \mathbf{D}_{\mu} X^{\rho}\right)+\left(X^{\rho} \mathbf{D}_{\alpha} \mathbf{R}_{\nu \mu \beta \rho}+\mathbf{R}_{\nu \mu \beta \rho} \mathbf{D}_{\alpha} X^{\rho}\right) \\
& +\left(\mathbf{R}_{\alpha \mu \nu \rho} \mathbf{D}^{\rho} X_{\beta}+\mathbf{R}_{\alpha \mu \beta \rho} \mathbf{D}_{\nu} X^{\rho}\right) .
\end{aligned}
$$

Therefore, replacing $\alpha \leftrightarrow \beta$ and $\mu \leftrightarrow \nu$,

$$
I I I=X^{\rho} \mathbf{D}_{\mu} \mathbf{R}_{\beta \alpha \nu \rho}+\mathbf{R}_{\beta \alpha \nu \rho} \mathbf{D}_{\mu} X^{\rho}
$$

and

$$
\begin{aligned}
I V & =\left(\mathbf{R}_{\mu \beta \nu \rho} \mathbf{D}^{\rho} X_{\alpha}+\mathbf{R}_{\mu \beta \alpha \rho} \mathbf{D}_{\nu} X^{\rho}\right)+\left(Z^{\rho} \mathbf{D}_{\beta} \mathbf{R}_{\mu \nu \alpha \rho}+\mathbf{R}_{\mu \nu \alpha \rho} \mathbf{D}_{\beta} X^{\rho}\right) \\
& +\left(\mathbf{R}_{\beta \nu \mu \rho} \mathbf{D}^{\rho} X_{\alpha}+\mathbf{R}_{\beta \nu \alpha \rho} \mathbf{D}_{\mu} X^{\rho}\right) .
\end{aligned}
$$


Therefore,

$$
\begin{aligned}
I+I I+ & I I I+I V=X^{\rho}\left(\mathbf{D}_{\nu} \mathbf{R}_{\alpha \beta \mu \rho}+\mathbf{D}_{\mu} \mathbf{R}_{\beta \alpha \nu \rho}+\mathbf{D}_{\alpha} \mathbf{R}_{\nu \mu \beta \rho}+\mathbf{D}_{\beta} \mathbf{R}_{\mu \nu \alpha \rho}\right) \\
& +\mathbf{D}_{\alpha} X^{\rho} \mathbf{R}_{\nu \mu \beta \rho}+\mathbf{D}_{\beta} X^{\rho} \mathbf{R}_{\mu \nu \alpha \rho} \\
& +\mathbf{D}^{\rho} X_{\alpha}\left(\mathbf{R}_{\mu \beta \nu \rho}+\mathbf{R}_{\beta \nu \mu \rho}\right)+\mathbf{D}^{\rho} X_{\beta}\left(\mathbf{R}_{\nu \alpha \mu \rho}+\mathbf{R}_{\alpha \mu \nu \rho}\right) \\
& +\mathbf{D}_{\mu} X^{\rho}\left(\mathbf{R}_{\nu \alpha \beta \rho}+\mathbf{R}_{\beta \alpha \nu \rho}+\mathbf{R}_{\beta \nu \alpha \rho}\right) \\
& +\mathbf{D}_{\nu} Z^{\rho}\left(\mathbf{R}_{\alpha \beta \mu \rho}+\mathbf{R}_{\alpha \mu \beta \rho}+\mathbf{R}_{\mu \beta \alpha \rho}\right) \\
& =2 X^{\rho} \mathbf{D}_{\rho} \mathbf{R}_{\alpha \beta \mu \nu}+2 \mathbf{D}_{\mu} X^{\rho} \mathbf{R}_{\alpha \beta \rho \nu}+2 \mathbf{D}_{\nu} X^{\rho} \mathbf{R}_{\alpha \beta \mu \rho} \\
& +2 \mathbf{D}_{\alpha} X^{\rho} \mathbf{R}_{\rho \beta \mu \nu}+2 \mathbf{D}_{\beta} X^{\rho} \mathbf{R}_{\alpha \rho \mu \nu}-{ }^{(X)} \pi_{\alpha}{ }^{\rho} \mathbf{R}_{\rho \beta \mu \nu}-{ }^{(X)} \pi_{\beta}{ }^{\rho} \mathbf{R}_{\alpha \rho \mu \nu} .
\end{aligned}
$$

The desired identity (2.15) follows.

Alternatively, one could also prove (2.15) by choosing a system of coordinates $x^{1}, \ldots, x^{d}$ such that $X=\partial_{d}$ and calculating explicitly ${ }^{(X)} \pi,{ }^{(X)} P$, and $\mathcal{L}_{X} \mathbf{R}$.

Finally, we derive a wave equation for the tensor $W$.

Lemma 2.9. With the notation in (2.7),

$$
\mathbf{D}^{\rho} \mathbf{D}_{\rho} W_{\alpha \beta \mu \nu}=\mathcal{M}(B, \mathbf{D} B, P, \mathbf{D} P, W)_{\alpha \beta \mu \nu} .
$$

Proof of Lemma 2.9. We use the identity

$$
\begin{aligned}
\mathbf{D}^{\sigma} \mathbf{D}_{\sigma} \mathbf{R}_{\alpha_{1} \alpha_{2} \alpha_{3} \alpha_{4}} & =\mathbf{R}_{\sigma \rho \alpha_{3} \alpha_{4}} \mathbf{R}^{\sigma \alpha_{1} \alpha_{2}}{ }^{\rho}+\mathbf{R}_{\sigma \alpha_{2} \rho \alpha_{4}} \mathbf{R}^{\sigma}{ }_{\alpha_{1} \alpha_{3}}{ }^{\rho}+\mathbf{R}_{\sigma \alpha_{2} \alpha_{3} \rho} \mathbf{R}^{\sigma}{ }_{\alpha_{1} \alpha_{4}}{ }^{\rho} \\
& -\mathbf{R}_{\sigma \rho \alpha_{3} \alpha_{4}} \mathbf{R}^{\sigma}{ }_{\alpha_{2} \alpha_{1}}{ }^{\rho}-\mathbf{R}_{\sigma \alpha_{1} \rho \alpha_{4}} \mathbf{R}^{\sigma}{ }_{\alpha_{2} \alpha_{3}}{ }^{\rho}-\mathbf{R}_{\sigma \alpha_{1} \alpha_{3} \rho} \mathbf{R}_{\alpha_{2} \alpha_{4}}{ }^{\rho},
\end{aligned}
$$

which is a well-known consequence of the Einstein vacuum equations. Using Lemma 2.2, we get

$$
\mathbf{D}_{\sigma}\left(\mathcal{L}_{Z} \mathbf{R}\right)_{\alpha_{1} \alpha_{2} \alpha_{3} \alpha_{4}}=\mathcal{L}_{Z}\left(\mathbf{D}_{\sigma} \mathbf{R}_{\alpha_{1} \alpha_{2} \alpha_{3} \alpha_{4}}\right)+\sum_{j=1}^{4} \Gamma_{\alpha_{j} \sigma \rho} \mathbf{R}_{\alpha_{1} \ldots}{ }^{\rho} \ldots \alpha_{4},
$$

and then

$$
\begin{aligned}
\square_{\mathbf{g}}\left(\mathcal{L}_{Z} \mathbf{R}\right)_{\alpha_{1} \alpha_{2} \alpha_{3} \alpha_{4}}= & \mathbf{D}^{\sigma} \mathcal{L}_{Z}\left(\mathbf{D}_{\sigma} \mathbf{R}_{\alpha_{1} \alpha_{2} \alpha_{3} \alpha_{4}}\right) \\
& +\sum_{j=1}^{4}\left[\left(\mathbf{D}^{\sigma} \Gamma_{\alpha_{j} \sigma \rho}\right) \mathbf{R}_{\alpha_{1} \ldots}{ }^{\rho} \ldots \alpha_{4}+\Gamma_{\alpha_{j} \sigma \rho} \mathbf{D}^{\sigma} \mathbf{R}_{\alpha_{1} \ldots}{ }^{\rho} \ldots \alpha_{4}\right]
\end{aligned}
$$

Therefore, after using Lemma 2.2 to commute derivatives again and (2.16), the equation for $\square_{\mathbf{g}}\left(\mathcal{L}_{Z} \mathbf{R}\right)$ above can be written, in schematic notation:

$$
\square_{\mathbf{g}}\left(\mathcal{L}_{Z} \mathbf{R}\right)_{\alpha_{1} \ldots \alpha_{4}}=\sum_{j=1}^{4}\left[\left(\mathbf{D}^{\sigma} \Gamma_{\alpha_{j} \sigma \rho}\right) \mathbf{R}_{\alpha_{1} \ldots}{ }^{\rho} \ldots \alpha_{4}\right]+\mathcal{M}\left(\pi, \mathbf{D} \pi,\left(\mathcal{L}_{Z} \mathbf{R}\right)\right)_{\alpha_{1} \ldots \alpha_{4}} .
$$

In view of the definition,

$$
\square_{\mathbf{g}}(B \odot \mathbf{R})_{\alpha_{1}, \ldots, \alpha_{4}}=\sum_{j=1}^{4} \mathbf{D}^{\sigma} \mathbf{D}_{\sigma} B_{\alpha_{j} \rho} \mathbf{R}_{\alpha_{1} \ldots}{ }^{\rho} \ldots \alpha_{4}+\mathcal{M}(B, \mathbf{D} B)_{\alpha_{1} \ldots \alpha_{4}} .
$$


Also using $\pi=\mathcal{M}(B), \mathbf{D} \pi=\mathcal{M}(\mathbf{D} B)$, and $\mathcal{L}_{Z} \mathbf{R}=\mathcal{M}(B, W)$, it follows that

$$
\square_{\mathbf{g}} W_{\alpha_{1} \ldots \alpha_{4}}=\sum_{j=1}^{4}\left[\mathbf{D}^{\sigma}\left(\Gamma_{\alpha_{j} \sigma \rho}-\mathbf{D}_{\sigma} B_{\alpha_{j} \rho}\right) \mathbf{R}_{\alpha_{1} \ldots}{ }^{\rho} \ldots \alpha_{4}\right]+\mathcal{M}(B, \mathbf{D} B, W)_{\alpha_{1} \ldots \alpha_{4}} .
$$

The lemma follows using the identity $\Gamma_{\alpha \beta \mu}-\mathbf{D}_{\beta} B_{\alpha \mu}=P_{\alpha \mu \beta}$.

We summarize some of the main results in this subsection in the following proposition:

Proposition 2.10. We assume that $O \subseteq \mathbf{M}, L, Z$ are as defined at the beginning of this section, and satisfy (2.3). In $\mathbf{M}$ we define

$$
\pi_{\alpha \beta}=\mathbf{D}_{\alpha} Z_{\beta}+\mathbf{D}_{\beta} Z_{\alpha} .
$$

We define the smooth antisymmetric tensor $\omega_{\alpha \beta}$ in $\mathbf{M}$ as the solution of the equation

$$
\mathbf{D}_{L} \omega_{\alpha \beta}=\pi_{\alpha \rho} \mathbf{D}_{\beta} L^{\rho}-\pi_{\beta \rho} \mathbf{D}_{\alpha} L^{\rho}, \quad \omega=0 \text { in } O .
$$

We also define the smooth tensors

$$
\begin{aligned}
& P_{\alpha \beta \mu}=(1 / 2)\left(\mathbf{D}_{\alpha} \pi_{\beta \mu}-\mathbf{D}_{\beta} \pi_{\alpha \mu}-\mathbf{D}_{\mu} \omega_{\alpha \beta}\right), \\
& B_{\alpha \beta}=(1 / 2)\left(\pi_{\alpha \beta}+\omega_{\alpha \beta}\right), \\
& W_{\alpha \beta \gamma \delta}=\left(\mathcal{L}_{Z} \mathbf{R}\right)_{\alpha \beta \gamma \delta}-(B \odot \mathbf{R})_{\alpha \beta \gamma \delta} .
\end{aligned}
$$

Then the following equations hold in $\mathbf{M}$ :

$$
\begin{aligned}
& \mathbf{D}^{\alpha} W_{\alpha \beta \gamma \delta}=\mathcal{M}(B, P, W)_{\beta \gamma \delta}, \\
& \mathbf{D}_{L} B=\mathcal{M}(B, P, W), \quad \mathbf{D}_{L} P=\mathcal{M}(B, P, W), \\
& \square W=\mathcal{M}(B, \mathbf{D} B, P, \mathbf{D} P, W, \mathbf{D} W),
\end{aligned}
$$

where $\mathcal{M}\left({ }^{(1)} B, \ldots,{ }^{(k)} B\right)$ is defined as in (2.7).

2.2. Carleman inequalities and the local extension theorem. Motivated by the identities summarized in Proposition 2.10, we consider solutions of systems of equations of the form

$$
\left\{\begin{array}{l}
\square_{\mathbf{g}} S=\mathcal{M}\left({ }^{(1)} B, \ldots,{ }^{(k)} B, S, \mathbf{D} S\right), \\
\mathbf{D}_{L}{ }^{(i)} B=\mathcal{M}\left({ }^{(1)} B, \ldots,{ }^{(k)} B, S, \mathbf{D} S\right), \quad i=1, \ldots, k .
\end{array}\right.
$$

We would like to prove that a solution $S,{ }^{(1)} B, \ldots,{ }^{(k)} B$ of such a system which vanishes on one side of a suitable hypersurface has to vanish in a neighborhood of the hypersurface. Such a result depends, of course, on convexity and non-degeneracy properties of the hypersurface. We recall (see Definition 1.1) that a domain $O$ is strongly pseudo-convex at a boundary point $p$ if there exists a defining function $f$ at $p, d f(p) \neq 0$ which verifies that

$$
\mathbf{D}^{2} f(X, X)(p)<0 \text { if } X \neq 0 \in T_{p}(\mathbf{M}) \text { satisfies } \mathbf{g}_{p}(X, X)=X(f)(p)=0 .
$$

We are now ready to prove Theorem 1.2 . We use the covariant equations derived in Proposition 2.10 (see (2.17)) and Carleman inequalities. We introduce a smooth system of coordinates $\Phi^{p}=\left(x^{1}, \ldots, x^{d}\right): B_{1} \rightarrow B_{1}(p), \Phi^{p}(0)=p$, where $B_{r}=\{x \in$ $\left.\mathbb{R}^{d}:|x|<r\right\}, r>0$, and $B_{1}(p)$ is an open neighborhood of $p$ in M. Let $\partial_{1}, \ldots, \partial_{d}$ denote the induced coordinate vector-fields in $B_{1}(p)$ and let $B_{r}(p)=\Phi^{p}\left(B_{r}\right), r \in$ 
$(0,1]$. For any smooth function $\phi: B \rightarrow \mathbb{C}$, where $B \subseteq B_{1}(p)$ is an open set, and $j=0,1, \ldots$, we define

$$
\left|\partial^{j} \phi(x)\right|=\sum_{\alpha_{1}, \ldots, \alpha_{j}=1}^{d}\left|\partial_{\alpha_{1}} \ldots \partial_{\alpha_{j}} \phi(x)\right|, \quad x \in B .
$$

We assume that

$$
\mathbf{g}_{\alpha \beta}(p)=\operatorname{diag}(-1, \ldots,-1,1, \ldots, 1) .
$$

We assume also that, for some constant $A \geq 1$,

$$
\sup _{x \in B_{1}(p)} \sum_{j=1}^{6} \sum_{\alpha, \beta=1}^{d}\left|\partial^{j} \mathbf{g}_{\alpha \beta}(x)\right|+\sup _{x \in B_{1}(p)} \sum_{j=1}^{4}\left|\partial^{j} f(x)\right| \leq A .
$$

We use the system of coordinates $\Phi^{p}$ in the neighborhood of the point $p$, and evaluate all the tensor-fields in the frame of coordinate vector-fields $\partial_{1}, \ldots, \partial_{d}$. In view of equations (2.17), for Theorem 1.2 it suffices to prove the following:

Lemma 2.11. Assume that $\delta_{0}>0$ and $G_{i}, H_{j}: B_{\delta_{0}}(p) \rightarrow \mathbb{C}$ are smooth functions, $i=1, \ldots, I, j=1, \ldots, J$, that satisfy the differential inequalities

$$
\left\{\begin{array}{l}
\left|\square_{\mathbf{g}} G_{i}\right| \leq M \sum_{l=1}^{I}\left(\left|G_{l}\right|+\left|\partial^{1} G_{l}\right|\right)+M \sum_{m=1}^{J}\left|H_{m}\right|, \\
\left|L\left(H_{j}\right)\right| \leq M \sum_{l=1}^{I}\left(\left|G_{l}\right|+\left|\partial^{1} G_{l}\right|\right)+M \sum_{m=1}^{J}\left|H_{m}\right|,
\end{array}\right.
$$

for any $i=1, \ldots, I, j=1, \ldots, J$, where $M \geq 1$ is a constant. Assume that $G_{i}=0$ and $H_{j}=0$ in $B_{\delta_{0}}(p) \cap O_{-}, i=1, \ldots, I, j=1, \ldots, J$. Assume also that $f$ is strongly pseudo-convex at $p$, in the sense of Definition 1.1, and $L(f)(p) \neq 0$. Then $G_{i}=0$ and $H_{j}=0$ in $B_{\delta_{1}}(p), i=1, \ldots, I, j=1, \ldots, J$, for some constant $\delta_{1} \in\left(0, \delta_{0}\right)$ sufficiently small.

Lemma 2.11 is proved in [1, Lemma 3.4], using two Carleman inequalities: Proposition 3.3 in [7] and Lemma A.3 in [1. The implicit constant $\delta_{1}>0$ depends only on constants $A$ in (2.21),$\delta_{0}$, and the constant $A_{1}$ in the following quantitative form of strong pseudo-convexity:

Lemma 2.12. (a) Assume that $f$ is strongly pseudo-convex at $p$. Then there are constants $A_{1} \geq A$ and $\mu \in\left[-A_{1}, A_{1}\right]$ such that, for any vector $X=X^{\alpha} \partial_{\alpha}$ at $p$,

$$
\begin{aligned}
& \left|\partial^{1} f(p)\right| \geq A_{1}^{-1}, \\
& X^{\alpha} X^{\beta}\left(\mu \mathbf{g}_{\alpha \beta}(p)-\mathbf{D}_{\alpha} \mathbf{D}_{\beta} f(p)\right)+A_{1}|X(f)(p)|^{2} \geq A_{1}^{-1}|X|^{2},
\end{aligned}
$$

where $|X|^{2}=\left(X^{1}\right)^{2}+\ldots+\left(X^{d}\right)^{2}$.

(b) Moreover, the inequalities (2.23) persist in a small neighborhood of $p$, in the sense that there is $\epsilon_{1}=\epsilon\left(A_{1}\right)>0$ such that for any vector-field $X=X^{\alpha} \partial_{\alpha}$ in $B_{\epsilon_{1}}(p)$, the inequalities

$$
\begin{aligned}
& \left|\partial^{1} f\right| \geq\left(2 A_{1}\right)^{-1}, \\
& X^{\alpha} X^{\beta}\left(\mu \mathbf{g}_{\alpha \beta}-\mathbf{D}_{\alpha} \mathbf{D}_{\beta} f\right)+A_{1}|X(f)|^{2} \geq\left(2 A_{1}\right)^{-1}|X|^{2},
\end{aligned}
$$

hold in $B_{\epsilon_{1}}(p)$, where $|X|^{2}=\left(X^{1}\right)^{2}+\ldots+\left(X^{d}\right)^{2}$ and $\mu$ is as in (2.23). 
Proof of Lemma 2.23. (a) The first inequality in (2.23) is just a quantitative form of the assumption that $p$ is not a critical point of $f$. To derive the second inequality, let $h_{\alpha \beta}=-\mathbf{D}_{\alpha} \mathbf{D}_{\beta} f(p)$ and

$$
\delta_{0}=\inf _{|X|=1, X^{\alpha} X_{\alpha}=X^{\alpha} \mathbf{D}_{\alpha} f=0} X^{\alpha} X^{\beta} h_{\alpha \beta} .
$$

By compactness, this infimum is attained, and it follows from (2.18) that $\delta_{0}>0$. By homogeneity, it follows that

$$
X^{\alpha} X^{\beta} h_{\alpha \beta} \geq \delta_{0}|X|^{2} \quad \text { if } X^{\alpha} X_{\alpha}=X^{\alpha} \mathbf{D}_{\alpha} f=0 .
$$

We would like to prove now that there is $n_{0} \in\{1,2, \ldots\}$ such that

$$
X^{\alpha} X^{\beta} h_{\alpha \beta}+n_{0}\left(X^{\alpha} \mathbf{D}_{\alpha} f(p)\right)^{2} \geq\left(\delta_{0} / 2\right)|X|^{2} \quad \text { if } X^{\alpha} X_{\alpha}=0 .
$$

Indeed, otherwise for any $n=1,2, \ldots$ there would exist a vector $X_{n}=X_{n}^{\alpha} \partial_{\alpha}$ such that $\left|X_{n}\right|=1, \mathbf{g}_{p}\left(X_{n}, X_{n}\right)=0$, and

$$
X_{n}^{\alpha} X_{n}^{\beta} h_{\alpha \beta}+n\left(X_{n}^{\alpha} \mathbf{D}_{\alpha} f(p)\right)^{2} \leq \delta_{0} / 2 .
$$

After passing to a subsequence, we may assume that $X_{n}$ converges to a vector $X$, with $|X|^{2}=1, X^{\alpha} X_{\alpha}=0, X^{\alpha} \mathbf{D}_{\alpha} f(p)=0$, and $X^{\alpha} X^{\beta} h_{\alpha \beta} \leq \delta_{0} / 2$, which contradicts (2.25). Therefore (2.26) holds for some constant $n_{0}$.

Let $\mathcal{C}_{+}=\left\{X \in T_{p} \mathbf{M}:|X|=1\right.$ and $\left.X^{\alpha} X_{\alpha}>0\right\}, \mathcal{C}_{-}=\left\{X \in T_{p} \mathbf{M}:|X|=\right.$ 1 and $\left.X^{\alpha} X_{\alpha}<0\right\}$ and, for $\delta \in[0,1], \mathcal{C}_{\delta}=\left\{X \in T_{p} \mathbf{M}:|X|=1\right.$ and $\left.\left|X^{\alpha} X_{\alpha}\right| \leq \delta\right\}$. Since the metric $\mathbf{g}$ is non-degenerate, we may assume that $\mathcal{C}_{+} \neq \emptyset$ (if $\mathcal{C}_{+}=\emptyset$, then $\mathcal{C}_{-} \neq \emptyset$ and the proof proceeds in a similar way). For $\rho \in \mathbb{R}$, we consider the function

$$
K_{\rho}: T_{p} \mathbf{M} \rightarrow \mathbb{R}, \quad K_{\rho}(X)=X^{\alpha} X^{\beta} h_{\alpha \beta}+n_{0}\left(X^{\alpha} \mathbf{D}_{\alpha} f(p)\right)^{2}+\rho X^{\alpha} X_{\alpha},
$$

where $n_{0}$ is as in (2.26). Using a simple compactness argument as before, it follows from (2.26), that

$$
\text { there is } \delta^{\prime}>0 \text { such that } K_{0}(X) \geq \delta_{0} / 4 \text { for any } X \in \mathcal{C}_{\delta^{\prime}} .
$$

Then it follows that there is $\rho_{1} \geq 0$ sufficiently large such that

$$
K_{\rho_{1}}(X) \geq 0 \text { if } X \in \mathcal{C}_{+} \text {and there is } X \in \mathcal{C}_{+} \text {such that } K_{-\rho_{1}}(X)<0 .
$$

Let

$$
\rho_{0}=\inf \left\{\rho \in\left[-\rho_{1}, \rho_{1}\right]: K_{\rho}(X) \geq 0 \text { for any } X \in \mathcal{C}_{+}\right\} .
$$

We analyze the function $K_{\rho_{0}}(X)=X^{\alpha} X^{\beta} k_{\alpha \beta}$, where

$$
k_{\alpha \beta}:=h_{\alpha \beta}+n_{0} \mathbf{D}_{\alpha} f(p) \mathbf{D}_{\beta} f(p)+\rho_{0} \mathbf{g}_{\alpha \beta} .
$$

In view of the definition of $\rho_{0}, K_{\rho_{0}}(X) \geq 0$ in $\mathcal{C}_{+}$. Moreover, using also (2.27), there exists $X_{0} \in \mathcal{C}_{+}$such that $K_{\rho_{0}}\left(X_{0}\right)=0$. Since $K_{\rho_{0}}$ is homogeneous of degree 2 , it follows that the point $X_{0}$ is a local minimum for $K_{\rho_{0}}$ in $T_{p} \mathbf{M}$. Therefore,

$$
V^{\alpha} X_{0}^{\beta} k_{\alpha \beta}=0 \text { and } V^{\alpha} V^{\beta} k_{\alpha \beta} \geq 0 \text { for any } V \in T_{p} \mathcal{O} \text {. }
$$

We show now that

$$
K_{\rho_{0}}(X) \neq 0 \text { for any } X \in \mathcal{C}_{-} .
$$

Indeed, assuming $K_{\rho_{0}}\left(X_{1}\right)=0$ for some $X_{1} \in \mathcal{C}_{-}$, it follows from (2.28) that $K_{\rho_{0}}\left(t X_{0}+(1-t) X_{1}\right)=0$ for any $t \in[0,1]$. However, this contradicts (2.27) since there is $t_{0} \in[0,1]$ such that $\mathbf{g}_{p}\left(t_{0} X_{0}+\left(1-t_{0}\right) X_{1}, t_{0} X_{0}+\left(1-t_{0}\right) X_{1}\right)=0$ and $t_{0} X_{0}+\left(1-t_{0}\right) X_{1} \neq 0$. 
Using (2.27), (2.28), and (2.29) it follows that $K_{\rho_{0}}(X)>0$ for any $x \in \mathcal{C}_{-} \cup \mathcal{C}_{\delta^{\prime \prime}}$, for some $\delta^{\prime \prime}>0$. A simple compactness argument then shows that there is $n_{1}$ large enough such that $K_{\rho_{0}+1 / n_{1}}>0$ in $\left\{X \in T_{p} \mathbf{M}:|X|=1\right\}$. The second inequality in (2.23) follows by setting $\mu=\rho_{0}+1 / n_{1}$ and $A_{1}$ sufficiently large.

Part (b) of the lemma follows from part (a) and the assumption (2.21).

\section{Proof of Theorem 1.3}

The plan of the proof is the following: we fix a point $p \in U_{0} \cap \mathcal{H}^{-} \cap \overline{\mathbf{E}}$, outside both the bifurcation sphere $S_{0}=\mathcal{H}^{-} \cap \mathcal{H}^{+}$and the axis of symmetry $\mathcal{A}=\{p \in \overline{\mathbf{E}}$ : $Z(p)=0\}$. Then we consider the Kerr metric $\mathbf{g}$ and the induced metric

$$
h_{\alpha \beta}=X \mathbf{g}_{\alpha \beta}-\mathbf{T}_{\alpha} \mathbf{T}_{\beta}, \quad \text { where } X=\mathbf{g}(\mathbf{T}, \mathbf{T}),
$$

on a hypersurface $\Pi$ passing through the point $p$ and transversal to $\mathbf{T}$. The metric $h$ is non-degenerate (Lorentzian) as long as $X>0$ in $\Pi$, which explains our assumption $0<a<m$. It is well known (see for example [12, Section 3]) that the Einstein vacuum equations together with stationarity $\mathcal{L}_{\mathbf{T}} \mathbf{g}=0$ are equivalent to the system of equations

$$
\begin{aligned}
& { }^{h} \mathbf{R i c}_{a b}=\frac{1}{2 X^{2}}\left(\nabla_{a} X \nabla_{b} X+\nabla_{a} Y \nabla_{b} Y\right), \\
& { }^{h} \square(X+i Y)=\frac{1}{X} h^{a b} \partial_{a}(X+i Y) \partial_{b}(X+i Y),
\end{aligned}
$$

in $\Pi$, where $Y$ is the Ernst potential associated to $\mathbf{T}$. We rederive these equations in Proposition 3.1 below, together with other explicit equations and identities that are needed for the proof of the theorem.

We then modify the metric $h$ and the functions $X$ and $Y$ in a neighborhood of the point $p$ in such a way that the identities (3.1) are still satisfied. The point is to prove the existence of a large family of smooth triplets $(\widetilde{h}, \widetilde{X}, \widetilde{Y})$ satisfying (3.1) and agreeing with the Kerr triplet in $\Pi \backslash \mathbf{E}$. This follows by solving a characteristic initial-value problem, using a generalization of the main theorem in [11.

Finally, in Proposition 3.5 we construct the space-time metric $\widetilde{\mathbf{g}}$,

$$
\widetilde{\mathbf{g}}_{a b}=\widetilde{X}^{-1} \widetilde{h}_{a b}+\widetilde{X} \widetilde{A}_{a} \widetilde{A}_{b}, \quad \widetilde{\mathbf{g}}_{a 4}=\widetilde{X} \widetilde{A}_{a}, \quad \widetilde{\mathbf{g}}_{44}=\widetilde{X}, \quad a, b=1,2,3,
$$

associated to the triplet $(\widetilde{h}, \widetilde{X}, \widetilde{Y})$, the vector-field $\mathbf{T}=\partial_{4}$, and a suitable 1 -form $\widetilde{A}$ which is defined in $\Pi$. By construction and [12, Theorem 1], this metric verifies the identities ${ }^{\mathbf{g}_{\mathbf{R i c}}}=0$ and $\mathcal{L}_{\mathbf{T}} \widetilde{\mathbf{g}}=0$, in a suitable open set $U$. Then we show that we have enough flexibility to choose initial conditions for $\widetilde{X}, \widetilde{Y}$ such that the vector-field $Z$ cannot be extended as a Killing vector-field for $\widetilde{\mathbf{g}}$ in the open set $U$.

3.1. Explicit calculations. We consider the Kerr space-time $\mathcal{K}(m, a)$ in standard Boyer-Lindquist coordinates,

$$
\mathbf{g}=-\frac{q^{2} \Delta}{\Sigma^{2}}(d t)^{2}+\frac{\Sigma^{2}(\sin \theta)^{2}}{q^{2}}\left(d \phi-\frac{2 a m r}{\Sigma^{2}} d t\right)^{2}+\frac{q^{2}}{\Delta}(d r)^{2}+q^{2}(d \theta)^{2}
$$

where

$$
\left\{\begin{array}{l}
\Delta=r^{2}+a^{2}-2 m r \\
q^{2}=r^{2}+a^{2}(\cos \theta)^{2}, \\
\Sigma^{2}=\left(r^{2}+a^{2}\right) q^{2}+2 m r a^{2}(\sin \theta)^{2}=\left(r^{2}+a^{2}\right)^{2}-a^{2}(\sin \theta)^{2} \Delta .
\end{array}\right.
$$


We make the change of variables:

$$
d u_{-}=d t-\left(r^{2}+a^{2}\right) \Delta^{-1} d r, \quad d \phi_{-}=d \phi-a \Delta^{-1} d r .
$$

In the new coordinates $\left(\theta, r, \phi_{-}, u_{-}\right)$the space-time metric becomes

$$
\begin{aligned}
& \mathbf{g}=q^{2} d \theta^{2}-\left(d u_{-} \otimes d r+d r \otimes d u_{-}\right)+a(\sin \theta)^{2}\left(d \phi_{-} \otimes d r+d r \otimes d \phi_{-}\right) \\
& -\frac{2 a m r(\sin \theta)^{2}}{q^{2}}\left(d \phi_{-} \otimes d u_{-}+d u_{-} \otimes d \phi_{-}\right)+\frac{\Sigma^{2}(\sin \theta)^{2}}{q^{2}} d \phi_{-}^{2}+\frac{2 m r-q^{2}}{q^{2}} d u_{-}^{2},
\end{aligned}
$$

and the vector-field $\mathbf{T}=d / d t$ becomes $\mathbf{T}=d / d u_{-}$. The metric $\mathbf{g}$ and the vectorfield $\mathbf{T}$ are smooth in the region

$$
R=\left\{\left(\theta, r, \phi_{-}, u_{-}\right) \in(0, \pi) \times(0, \infty) \times(-\pi, \pi) \times \mathbb{R}: 2 m r-q^{2}>0\right\} .
$$

Let

and

$$
X=\mathbf{g}(\mathbf{T}, \mathbf{T})=\frac{2 m r-q^{2}}{q^{2}}, \quad h_{\alpha \beta}=X \mathbf{g}_{\alpha \beta}-\mathbf{T}_{\alpha} \mathbf{T}_{\beta}
$$

$$
\Pi=\left\{\left(\theta, r, \phi_{-}, u_{-}\right) \in R: u_{-}=0\right\} .
$$

Let

$$
\partial_{1}=\frac{d}{d \theta}, \quad \partial_{2}=\frac{d}{d r}, \quad \partial_{3}=\frac{d}{d \phi_{-}}
$$

denote the vector-fields in $\Pi$ induced by coordinates $\left(\theta, r, \phi_{-}\right)$. We calculate the components of the metric $h$ along the surface $\Pi$,

$$
\begin{array}{ll}
h_{11}=2 m r-q^{2}, & h_{12}=0, \quad h_{13}=0, \quad h_{22}=-1, \\
h_{23}=-a(\sin \theta)^{2}, & h_{33}=-\Delta(\sin \theta)^{2} .
\end{array}
$$

Therefore,

$$
\begin{aligned}
h^{11} & =\frac{1}{2 m r-q^{2}}, & h^{12} & =0, \quad h^{13}=0, \quad h^{22}=\frac{\Delta}{2 m r-q^{2}}, \\
h^{23} & =\frac{-a}{2 m r-q^{2}}, & h^{33} & =\frac{1}{(\sin \theta)^{2}\left(2 m r-q^{2}\right)} .
\end{aligned}
$$

Let

$$
\Gamma_{c a b}=h\left(\nabla_{\partial_{b}} \partial_{a}, \partial_{c}\right)=(1 / 2)\left(\partial_{a} h_{b c}+\partial_{b} h_{a c}-\partial_{c} h_{a b}\right), \quad \Gamma_{a b}^{d}=h^{c d} \Gamma_{c a b} .
$$

Using (3.6) and (3.7) we calculate the following:

$$
\begin{aligned}
& \Gamma_{11}^{1}=\frac{a^{2} \sin \theta \cos \theta}{2 m r-q^{2}}, \quad \Gamma_{11}^{2}=\frac{\Delta(r-m)}{2 m r-q^{2}}, \quad \Gamma^{3}{ }_{11}=\frac{a(m-r)}{2 m r-q^{2}}, \\
& \Gamma_{12}^{1}=\frac{m-r}{2 m r-q^{2}}, \quad \Gamma_{12}^{2}=\frac{a^{2} \sin \theta \cos \theta}{2 m r-q^{2}}, \quad \Gamma^{3}{ }_{12}=\frac{-a \cot \theta}{2 m r-q^{2}}, \\
& \Gamma_{13}^{1}=0, \quad \Gamma_{13}^{2}=0, \quad \Gamma^{3}{ }_{13}=\cot \theta, \\
& \Gamma_{22}^{1}=0, \quad \Gamma_{22}^{2}=0, \quad \Gamma_{22}^{3}=0, \\
& \Gamma_{23}^{1}=\frac{a \sin \theta \cos \theta}{2 m r-q^{2}}, \quad \Gamma_{23}^{2}=\frac{a(r-m)(\sin \theta)^{2}}{2 m r-q^{2}}, \quad \Gamma_{23}^{3}=\frac{m-r}{2 m r-q^{2}}, \\
& \Gamma_{33}^{1}=\frac{\Delta \sin \theta \cos \theta}{2 m r-q^{2}}, \quad \Gamma_{33}^{2}=\frac{\Delta(r-m)(\sin \theta)^{2}}{2 m r-q^{2}},
\end{aligned}
$$


We have

$$
\begin{aligned}
{ }^{h} \mathbf{R}\left(\partial_{a}, \partial_{c}\right) \partial_{b} & =\nabla_{\partial_{a}}\left(\nabla_{\partial_{c}} \partial_{b}\right)-\nabla_{\partial_{c}}\left(\nabla_{\partial_{a}} \partial_{b}\right) \\
& =\nabla_{\partial_{a}}\left(\Gamma_{b c}^{d} \partial_{d}\right)-\nabla_{\partial_{c}}\left(\Gamma_{b a}^{d} \partial_{d}\right) \\
& =\partial_{a}\left(\Gamma_{b c}^{d}\right) \partial_{d}+\Gamma_{b c}^{d} \Gamma_{d a}^{e} \partial_{e}-\partial_{c}\left(\Gamma_{b a}^{d}\right) \partial_{d}+\Gamma_{b a}^{d} \Gamma_{d c}^{e} \partial_{e} \\
& =\left[\partial_{a}\left(\Gamma_{b c}^{d}\right)-\partial_{c}\left(\Gamma_{b a}^{d}\right)+\Gamma_{b c}^{e} \Gamma_{e a}^{d}-\Gamma_{b a}^{e} \Gamma_{e c}^{d} \partial_{d} .\right.
\end{aligned}
$$

Therefore,

$$
{ }^{h} \mathbf{R i c}_{b a}=\partial_{c}\left(\Gamma_{b a}^{c}\right)-\partial_{a}\left(\Gamma_{b c}^{c}\right)+\Gamma_{b a}^{d} \Gamma_{d c}^{c}-\Gamma_{c b}^{d} \Gamma_{d a}^{c} .
$$

Using (3.8) we calculate

$$
\Gamma_{b c}^{c}=(1 / 2) h^{c a}\left(\partial_{b} h_{c a}\right)=(1 / 2) \partial_{b}(\log |h|)=\partial_{b}\left(\log \left(\sin \theta\left(2 m r-q^{2}\right)\right)\right) .
$$

Thus,

$$
\begin{aligned}
& { }^{h} \mathbf{R i c}_{11}=\frac{2 m^{2} a^{2}(\sin \theta)^{2}}{\left(2 m r-q^{2}\right)^{2}}, \quad{ }^{h} \mathbf{R i c}_{12}=0, \quad{ }^{h} \mathbf{R i c}_{13}=0 \\
& { }^{h} \mathbf{R i c}_{22}=\frac{2 m^{2}}{\left(2 m r-q^{2}\right)^{2}}, \quad{ }^{h} \mathbf{R i c}_{23}=0, \quad{ }^{h} \mathbf{R i c}_{33}=0 .
\end{aligned}
$$

Let

$$
\begin{aligned}
& X=\frac{2 m r-q^{2}}{q^{2}}, \quad Y=-\frac{2 m a \cos \theta}{q^{2}}, \\
& T_{a b}=\frac{1}{2 X^{2}}\left(\nabla_{a} X \nabla_{b} X+\nabla_{a} Y \nabla_{b} Y\right) .
\end{aligned}
$$

We calculate

$$
\begin{aligned}
& \partial_{1} X=\frac{4 a^{2} m r \sin \theta \cos \theta}{q^{4}}, \quad \partial_{2} X=\frac{2 m q^{2}-4 m r^{2}}{q^{4}}, \quad \partial_{3} X=0, \\
& \partial_{1} Y=\frac{2 m a \sin \theta q^{2}-4 m a^{3} \sin \theta(\cos \theta)^{2}}{q^{4}}, \quad \partial_{2} Y=\frac{4 m r a \cos \theta}{q^{4}}, \quad \partial_{3} Y=0 .
\end{aligned}
$$

Therefore,

$$
\begin{aligned}
& T_{11}=\frac{2 m^{2} a^{2}(\sin \theta)^{2}}{\left(2 m r-q^{2}\right)^{2}}, \quad T_{12}=0, \quad T_{13}=0, \\
& T_{22}=\frac{2 m^{2}}{\left(2 m r-q^{2}\right)^{2}}, \quad T_{23}=0, \quad T_{33}=0 .
\end{aligned}
$$

Also using (3.12) it follows that

$$
{ }^{h} \mathbf{R i c}=T .
$$

Using (3.7), (3.14), and $|h|=(\sin \theta)^{2}\left(2 m r-q^{2}\right)^{2}$ we calculate

$$
\begin{aligned}
|h|^{1 / 2} h^{1 j} \partial_{j}(X+i Y) & =i \frac{2 a m(\sin \theta)^{2}}{q^{4}}(r-i a \cos \theta)^{2}, \\
|h|^{1 / 2} h^{2 j} \partial_{j}(X+i Y) & =\frac{-2 m \Delta \sin \theta}{q^{4}}(r-i a \cos \theta)^{2}, \\
|h|^{1 / 2} h^{3 j} \partial_{j}(X+i Y) & =\frac{2 m a \sin \theta}{q^{4}}(r-i a \cos \theta)^{2} .
\end{aligned}
$$


Therefore

$$
\begin{aligned}
{ }^{h} \square X & =\frac{24 m^{2} r^{2} a^{2}(\cos \theta)^{2}-4 m^{2} r^{4}-4 m^{2} a^{4}(\cos \theta)^{4}}{q^{6}\left(2 m r-q^{2}\right)}, \\
{ }^{h} \square Y & =\frac{16 m^{2} r a \cos \theta\left(r^{2}-a^{2}(\cos \theta)^{2}\right)}{q^{6}\left(2 m r-q^{2}\right)} .
\end{aligned}
$$

We also calculate

$$
\begin{aligned}
& X^{-1} h^{i j}\left(\partial_{i} X \partial_{j} X-\partial_{i} Y \partial_{j} Y\right)=\frac{24 m^{2} r^{2} a^{2}(\cos \theta)^{2}-4 m^{2} r^{4}-4 m^{2} a^{4}(\cos \theta)^{4}}{q^{6}\left(2 m r-q^{2}\right)}, \\
& 2 X^{-1} h^{i j} \partial_{i} X \partial_{j} Y=\frac{16 m^{2} r a \cos \theta\left(r^{2}-a^{2}(\cos \theta)^{2}\right)}{q^{6}\left(2 m r-q^{2}\right)} .
\end{aligned}
$$

Therefore,

$$
\begin{aligned}
& { }^{h} \mathbf{R i c}_{a b}=\frac{1}{2 X^{2}}\left(\nabla_{a} X \nabla_{b} X+\nabla_{a} Y \nabla_{b} Y\right), \\
& { }^{h} \square(X+i Y)=\frac{1}{X} h^{a b} \partial_{a}(X+i Y) \partial_{b}(X+i Y) .
\end{aligned}
$$

The components of the spacetime metric $\mathbf{g}$ in the coordinates $\left(\theta, r, \phi_{-}, u_{-}\right)$(see (3.4) ) have the form

$$
\mathbf{g}_{a b}=X^{-1} h_{a b}+X A_{a} A_{b}, \quad \mathbf{g}_{a 4}=X A_{a}, \quad \mathbf{g}_{44}=X, \quad a, b=1,2,3 .
$$

or, with $x=(\theta, r, \phi)$,

$$
\mathbf{g}=\left(X d u_{-}+A_{a} d x^{a}\right)^{2}+X^{-1} h_{a b} d x^{a} d x^{b},
$$

where

$$
A_{1}=0, \quad A_{2}=-\frac{q^{2}}{2 m r-q^{2}}, \quad A_{3}=-\frac{2 a m r(\sin \theta)^{2}}{2 m r-q^{2}} .
$$

We compute

$$
\begin{aligned}
\partial_{1} A_{2}-\partial_{2} A_{1} & =\frac{4 a^{2} m r \sin \theta \cos \theta}{\left(2 m r-q^{2}\right)^{2}} \\
\partial_{2} A_{3}-\partial_{3} A_{2} & =\frac{-2 m a(\sin \theta)^{2}\left(r^{2}-a^{2}(\cos \theta)^{2}\right)}{\left(2 m r-q^{2}\right)^{2}}, \\
\partial_{3} A_{1}-\partial_{1} A_{3} & =\frac{-4 m r a \Delta \sin \theta \cos \theta}{\left(2 m r-q^{2}\right)^{2}} .
\end{aligned}
$$

Therefore, also using (3.15), with ${ }^{h} \in_{123}=-|h|^{1 / 2}$, gives us

$$
X^{2}\left(\nabla_{a} A_{b}-\nabla_{b} A_{a}\right)={ }^{h} \in_{a b c} \nabla^{c} Y .
$$

To summarize, we verified the following:

Proposition 3.1. With the notation above, the metric $h$, the functions $X, Y$, and the 1-form A satisfy the identities (in $\Pi$ )

$$
\begin{aligned}
& { }^{h} \mathbf{R i c}_{a b}=\frac{1}{2 X^{2}}\left(\nabla_{a} X \nabla_{b} X+\nabla_{a} Y \nabla_{b} Y\right), \\
& { }^{h} \square(X+i Y)=\frac{1}{X} h^{a b} \partial_{a}(X+i Y) \partial_{b}(X+i Y), \\
& X^{2}\left(\nabla_{a} A_{b}-\nabla_{b} A_{a}\right)={ }^{h} \in_{a b c} \nabla^{c} Y .
\end{aligned}
$$


Remark 3.2. Under a change of coordinates of the form $u_{-}^{\prime}=u_{-}-f\left(x^{1}, x^{2}, x^{3}\right)$ the 1-form $A=A_{a} d x^{a}$ changes according to the formula $A^{\prime}=A-d f$. The change of coordinates amounts to a choice of the hypersurface $\Pi$, i.e., instead of $u_{-}=0$ we would choose $u_{-}=f(\theta, r, \phi)$.

3.2. The metric $\tilde{h}$. We would like to construct now a large family of triplets $(\widetilde{h}, \widetilde{X}, \widetilde{Y})$ and 1-forms $\widetilde{A}$, such that the identities in Proposition 3.1 are still satisfied in a neighborhood in $\Pi$ of a fixed point $p \in\left(U_{0} \cap \mathcal{H}^{-} \cap \overline{\mathbf{E}}\right) \backslash\left(\mathcal{A} \cup S_{0}\right)$. Let

$$
\mathcal{N}_{0}=\left\{x \in \Pi: r(x)=r_{+}:=m+\sqrt{m^{2}-a^{2}}\right\} .
$$

This is a 2-dimensional hypersurface in $\Pi$; the vector-fields $\partial_{1}$ and $\partial_{3}$ are tangent to $\mathcal{N}_{0}$ and, using (3.6) and (3.9),

$$
h\left(\partial_{3}, \partial_{3}\right)=h\left(\partial_{3}, \partial_{1}\right)=0, \quad \nabla_{\partial_{3}} \partial_{3}=-\left[(m / a)^{2}-1\right]^{1 / 2} \partial_{3}, \quad \text { along } \mathcal{N}_{0} .
$$

Therefore, $\mathcal{N}_{0}$ is a null hypersurface in $\Pi$. Along $\mathcal{N}_{0} \subset \Pi$ we define the smooth, transversal, null vector-field,

$$
L=\left(2 a^{2}(\sin \theta)^{2}-\Delta\right)^{-1} \cdot\left[2 a \partial_{2}-(\sin \theta)^{-2} \partial_{3}\right] .
$$

Using (3.6), it follows that

$$
h(L, L)=h\left(L, \partial_{1}\right)=0, \quad\left[L, \partial_{3}\right]=0, \quad h\left(L, \partial_{3}\right)=-1, \quad \text { along } \mathcal{N}_{0} .
$$

Let

$$
P=\left\{x \in \mathcal{N}_{0}: \phi_{-}(x)=0\right\}, \quad p=\left\{x \in P: \theta(x)=\theta_{0} \in(0, \pi)\right\} .
$$

Thus $P$ is a 1 -dimensional smooth curve in $\mathcal{N}_{0}$ and $p \in P$ is a point. We extend the vector-field $L$ to a small open neighborhood $D$ of $p$ in $\Pi$, by solving the geodesic equation

$$
\nabla_{L} L=0 \quad \text { in } D \text {. }
$$

Then we construct the null hypersurface $\mathcal{N}_{1}$ in $D$ as the congruence of geodesic curves tangent to $L$ and passing through the curve $P$. We also fix a time-orientation in $D$ such that $\partial_{3}$ and $L$ are future-directed null vector-fields along $P \cap D$, and we let $J^{+}\left(\mathcal{N}_{1}\right)$ denote the causal future of $\mathcal{N}_{1}$ in $D$. Let

$$
D_{-}=\{x \in D: \Delta(x)<0\}, \quad D_{+}=\{x \in D: \Delta(x)>0\} .
$$

The following proposition is a consequence of a more general version 6 of the main theorem in [1].

Proposition 3.3. Assume $\widetilde{X}, \widetilde{Y}: \mathcal{N}_{1} \rightarrow \mathbb{R}$ are smooth functions satisfying

$$
\widetilde{X}=X \text { and } \tilde{Y}=Y \text { in } \mathcal{N}_{1} \cap D_{-} .
$$

Then there is a small neighborhood $D^{\prime}$ of $p$ in $\Pi$, a smooth metric $\widetilde{h}$ in $J^{+}\left(\mathcal{N}_{1}\right) \cap D^{\prime}$, and smooth extensions $\widetilde{X}, \widetilde{Y}: J^{+}\left(\mathcal{N}_{1}\right) \cap D^{\prime} \rightarrow \mathbb{R}$ such that, in $J^{+}\left(\mathcal{N}_{1}\right) \cap D^{\prime}$,

$$
\begin{aligned}
& { }^{\widetilde{h}} \mathbf{R i c}_{a b}=\frac{1}{2 \widetilde{X}^{2}}\left(\nabla_{a} \tilde{X} \nabla_{b} \tilde{X}+\nabla_{a} \widetilde{Y} \nabla_{b} \tilde{Y}\right), \\
& { }^{\widetilde{h}} \square(\widetilde{X}+i \widetilde{Y})=\frac{1}{\widetilde{X}} \widetilde{h}^{a b} \partial_{a}(\widetilde{X}+i \widetilde{Y}) \partial_{b}(\widetilde{X}+i \widetilde{Y}) .
\end{aligned}
$$

\footnotetext{
${ }^{6}$ The result in 11 is stated only for the Einstein vacuum equations. It is clear, nevertheless, that a straightforward generalization of it applies to the coupled Einstein Wave Map equations in any dimension. Note that in our special case of $2+1$ dimensions the only dynamical variables are $X$ and $Y$, corresponding to the wave map. We note also the more recent work of Luk [9], which is closer to our setting; in particular, it justifies the second identity in 3.24.
} 
In addition,

$$
\widetilde{X}=X, \quad \widetilde{Y}=Y, \quad \widetilde{h}=h \quad \text { in } J^{+}\left(\mathcal{N}_{1}\right) \cap D^{\prime} \cap D_{-},
$$

and, for any vector-field $V$ tangent to $\mathcal{N}_{1} \cap D^{\prime}$,

$$
\widetilde{h}(L, V)=0 \text { and } \widetilde{\nabla}_{L} L=0 \quad \text { along } \mathcal{N}_{1} \cap D^{\prime} .
$$

To be able to construct the desired space-time metric $\widetilde{\mathbf{g}}$ we also need to extend the 1 -form $A$ (compare with the formula (3.30) ). More precisely:

Proposition 3.4. There is a smooth 1 -form $\widetilde{A}_{a}$ in a neighborhood $\mathcal{D}$ of $p$ in $J^{+}\left(\mathcal{N}_{1}\right)$ satisfying (compare with (3.19)

$$
\begin{aligned}
& \widetilde{X}^{2}\left(\widetilde{\nabla}_{a} \widetilde{A}_{b}-\widetilde{\nabla}_{b} \widetilde{A}_{a}\right)=\widetilde{\epsilon}_{a b c} \widetilde{\nabla}^{c} \widetilde{Y}, \\
& \widetilde{A}=A \quad \text { in } \mathcal{D} \cap D_{-} .
\end{aligned}
$$

Proof of Proposition 3.4 . Let $\widetilde{L}$ denote the geodesic vector-field (i.e., $\widetilde{\nabla} \widetilde{L} \widetilde{L}=0$ ) generated in a neighborhood of the point $p$ in the vector-field $L$ defined on $\mathcal{N}_{0}$ in (3.20), so

$$
\widetilde{L}=L \text { in }\left[\left(J^{+}\left(\mathcal{N}_{1}\right) \cap D_{-}\right) \cup \mathcal{N}_{1}\right] \cap D^{\prime} .
$$

Let $g=L^{a} A_{a}$, defined using the unperturbed $L$ and $A$. We then define the form $\widetilde{A}$ as the solution of the transport equation, in a neighborhood of the point $p$ in $J^{+}\left(\mathcal{N}_{1}\right)$

$$
\begin{aligned}
& \widetilde{L}^{a} \widetilde{\nabla}_{a} \widetilde{A}_{b}+\widetilde{A}_{a} \widetilde{\nabla}_{b} \widetilde{L}^{a}=\widetilde{\epsilon}_{a b c} \widetilde{X}^{-2} \widetilde{L}^{a} \widetilde{\nabla}^{c} \widetilde{Y}+\widetilde{\nabla}_{b} g, \\
& \widetilde{A}=A \quad \text { along } \mathcal{N}_{0} .
\end{aligned}
$$

It follows easily from (3.19) that the form $A$ verifies this transport equation in $D_{-}$, thus $\widetilde{A}$ is a well-defined smooth form in a neighborhood $\mathcal{D}$ of $p$ in $J^{+}\left(\mathcal{N}_{1}\right)$ and $\widetilde{A}=A$ in $D_{-}$. It remains to prove the identity in the first line of (3.25). We observe first that $\widetilde{A}_{a} \widetilde{L}^{a}=g$ in a neighborhood of $p$ in $J^{+}\left(\mathcal{N}_{1}\right)$. Indeed, using the definition (3.26) we compute

$$
\widetilde{L}^{a} \widetilde{\nabla}_{a}\left(\widetilde{L}^{b} \widetilde{A}_{b}-g\right)=\widetilde{L}^{b} \widetilde{L}^{a} \widetilde{\nabla}_{a} \widetilde{A}_{b}-\widetilde{L}^{a} \widetilde{\nabla}_{a} g=0,
$$

therefore,

$$
\widetilde{L}^{b} \widetilde{A}_{b}=g \quad \text { in a neighborhood of } p \text { in } J^{+}\left(\mathcal{N}_{1}\right)
$$

Letting

$$
\widetilde{Q}_{a b}=\left(\widetilde{\nabla}_{a} \widetilde{A}_{b}-\widetilde{\nabla}_{b} \widetilde{A}_{a}\right)-\widetilde{X}^{-2} \widetilde{\epsilon}_{a b c} \widetilde{\nabla}^{c} \widetilde{Y},
$$

it follows from (3.26) and (3.27) that

$$
\widetilde{L}^{a} \widetilde{Q}_{a b}=0, \quad \widetilde{Q}_{a b}+\widetilde{Q}_{b a}=0 .
$$

To show that $\widetilde{Q}$ vanish identically we derive a transport equation for it. Using Lemma 2.2 and the identity (3.26) in the form $\mathcal{L}_{\widetilde{L}^{2}} \widetilde{A}_{c}=\widetilde{\epsilon}_{m c n} \widetilde{X}^{-2} \widetilde{L}^{m} \widetilde{\nabla}^{n} \widetilde{Y}+\widetilde{\nabla}_{c} g$, we calculate

$$
\widetilde{X}^{2} \mathcal{L}_{\widetilde{L}} \widetilde{Q}_{a b}=\widetilde{E}_{a b}
$$


where

$$
\begin{aligned}
& \widetilde{E}_{a b}=\widetilde{X}^{2}\left(\widetilde{\nabla}_{a} \mathcal{L}_{\widetilde{L}} \widetilde{A}_{b}-\widetilde{\nabla}_{b} \mathcal{L}_{\widetilde{L}} \widetilde{A}_{a}\right)+2 \widetilde{X}^{-1} \widetilde{L}(\widetilde{X}) \widetilde{\epsilon}_{a b m} \widetilde{\nabla}^{m} \widetilde{Y} \\
& -\widetilde{\epsilon}_{a b m} \widetilde{\nabla}^{m} \tilde{Y} \widetilde{\nabla}^{n} \widetilde{L}_{n}-\widetilde{\epsilon}_{a b m} \mathcal{L}_{\widetilde{L}}\left(\widetilde{\nabla}^{m} \tilde{Y}\right) \\
& =\widetilde{\epsilon}_{m b n} \widetilde{\nabla}_{a} \widetilde{L}^{m} \widetilde{\nabla}^{n} \widetilde{Y}+\widetilde{\epsilon}_{m b n} \widetilde{L}^{m} \widetilde{\nabla}_{a} \widetilde{\nabla}^{n} \widetilde{Y}-2 \widetilde{X}^{-1} \widetilde{\nabla}_{a}(\widetilde{X}) \widetilde{\epsilon}_{m b n} \widetilde{L}^{m} \widetilde{\nabla}^{n} \widetilde{Y} \\
& -\widetilde{\epsilon}_{m a n} \widetilde{\nabla}_{b} \widetilde{L}^{m} \widetilde{\nabla}^{n} \widetilde{Y}-\widetilde{\epsilon}_{m a n} \widetilde{L}^{m} \widetilde{\nabla}_{b} \widetilde{\nabla}^{n} \widetilde{Y}+2 \widetilde{X}^{-1} \widetilde{\nabla}_{b}(\widetilde{X}) \widetilde{\epsilon}_{m a n} \widetilde{L}^{m} \widetilde{\nabla}^{n} \widetilde{Y} \\
& +2 \widetilde{X}^{-1} \widetilde{L}(\widetilde{X}) \widetilde{\epsilon}_{a b m} \widetilde{\nabla}^{m} \widetilde{Y}-\widetilde{\epsilon}_{a b m} \widetilde{\nabla}^{m} \widetilde{Y} \widetilde{\nabla}^{n} \widetilde{L}_{n}-\widetilde{\epsilon}_{a b m}\left(\widetilde{L}^{n} \widetilde{\nabla}_{n} \widetilde{\nabla}^{m} \widetilde{Y}-\widetilde{\nabla}^{n} \widetilde{Y} \widetilde{\nabla}_{n} \widetilde{L}^{m}\right) \text {. }
\end{aligned}
$$

Now we calculate

$$
\begin{aligned}
\widetilde{\epsilon}^{a b c} \widetilde{E}_{a b} & =2 \widetilde{\nabla}^{c} \widetilde{Y} \widetilde{\nabla}^{n} \widetilde{L}_{n}-2 \widetilde{\nabla}^{n} \widetilde{Y} \widetilde{\nabla}_{n} \widetilde{L}^{c}+2 \widetilde{L}^{m} \widetilde{\nabla}_{m} \widetilde{\nabla}^{c} \widetilde{Y}-2 \widetilde{L}^{c} \widetilde{\nabla}_{n} \widetilde{\nabla}^{n} \widetilde{Y} \\
& -4 \widetilde{X}^{-1} \widetilde{\nabla}_{m}(\widetilde{X}) \widetilde{L}^{m} \widetilde{\nabla}^{c} \widetilde{Y}+4 \widetilde{X}^{-1} \widetilde{\nabla}_{n}(\widetilde{X}) \widetilde{L}^{c} \widetilde{\nabla}^{n} \widetilde{Y} \\
& +4 \widetilde{X}^{-1} \widetilde{L}(\widetilde{X}) \widetilde{\nabla}^{c} \widetilde{Y}-2 \widetilde{\nabla}^{c} \widetilde{Y} \widetilde{\nabla}^{n} \widetilde{L}_{n}-2 \widetilde{L}^{n} \widetilde{\nabla}_{n} \widetilde{\nabla}^{c} \widetilde{Y}+2 \widetilde{\nabla}^{n} \widetilde{Y} \widetilde{\nabla}_{n} \widetilde{L}^{c} \\
& =-2 \widetilde{L}^{c} \widetilde{\nabla}_{n} \widetilde{\nabla}^{n} \widetilde{Y}+4 \widetilde{L}^{c} \widetilde{X}^{-1} \widetilde{\nabla}_{n}(\widetilde{X}) \widetilde{\nabla^{n}} \widetilde{Y}
\end{aligned}
$$

Since $\widetilde{\nabla}_{n} \widetilde{\nabla}^{n} \widetilde{Y}=2 \widetilde{X}^{-1} \widetilde{\nabla}_{n}(\widetilde{X}) \widetilde{\nabla}^{n} \widetilde{Y}$ (see (3.22) ), it follows that $\widetilde{\epsilon}^{a b c} \widetilde{E}_{a b}=0$. Therefore, $\widetilde{E}_{a b}=0$. Since $\widetilde{Q}=0$ in $\mathcal{D} \cap D_{-}$it follows that $\widetilde{Q}=0$ in $\mathcal{D}$ as desired.

3.3. The space-time metric. Let $\widetilde{X}, \widetilde{Y}, \widetilde{h}, \mathcal{D}$, and $\widetilde{A}$ be as before. In $\mathcal{D} \times I$, where $I \subset \mathbb{R}$ is an open interval, we define the Lorentz metric $\widetilde{\mathbf{g}}$ by

$$
\widetilde{\mathbf{g}}_{a b}=\widetilde{X}^{-1} \widetilde{h}_{a b}+\widetilde{X} \widetilde{A}_{a} \widetilde{A}_{b}, \quad \widetilde{\mathbf{g}}_{a 4}=\widetilde{X} \widetilde{A}_{a}, \quad \widetilde{\mathbf{g}}_{44}=\widetilde{X}, \quad a, b=1,2,3 .
$$

The functions $\widetilde{X}, \widetilde{Y}, \widetilde{A}_{a}, \widetilde{h}_{a b}$, originally defined in $\mathcal{D}$ are extended to $\mathcal{D} \times I$ by

$$
\partial_{4}(\widetilde{X})=\partial_{4}(\widetilde{Y})=\partial_{4}\left(\widetilde{A}_{a}\right)=\partial_{4}\left(\widetilde{h}_{a b}\right)=0, \quad a, b=1,2,3 .
$$

Using (3.30), it follows that, with $\widetilde{A}^{a}=\widetilde{h}^{a b} \widetilde{A}_{b}, a=1,2,3$,

$$
\widetilde{\mathbf{g}}^{a b}=\widetilde{X} \widetilde{h}^{a b}, \quad \widetilde{\mathbf{g}}^{a 4}=-\widetilde{X} \widetilde{A}^{a}, \quad \widetilde{\mathbf{g}}^{44}=\widetilde{X}^{-1}+\widetilde{X} \widetilde{A}^{a} \widetilde{A}_{a}, \quad|\widetilde{\mathbf{g}}|=\widetilde{X}^{-2}|\widetilde{h}| .
$$

Proposition 3.5. (a) The metric $\widetilde{\mathbf{g}}$ agrees with the Kerr metric $\mathbf{g}$ in $\left(\mathcal{D} \cap D_{-}\right) \times I$ and satisfies

$$
\mathcal{L}_{\partial_{4}} \widetilde{\mathbf{g}}=0, \quad \widetilde{\mathbf{g}}_{\mathbf{R i c}}=0 \quad \text { in } \mathcal{D} \times \mathbb{R} .
$$

(b) If $Z=Z^{4} \partial_{4}+Z^{a} \partial_{a}$ is a Killing vector-field for $\widetilde{\mathbf{g}}$ in $\mathcal{D} \times I$ and if $\left[Z, \partial_{4}\right]=0$ in $\mathcal{D} \times I$, then $Z^{\prime}=Z^{a} \partial_{a}$ is a Killing vector-field for $\widetilde{h}$ in $\mathcal{D}$ satisfying $Z^{\prime}(\widetilde{X})=$ $Z^{\prime}(\tilde{Y})=0$, i.e.,

$$
Z^{\prime}(\widetilde{X})=Z^{\prime}(\tilde{Y})=0, \quad\left(\mathcal{L}_{Z^{\prime}}, \widetilde{h}\right)_{a b}=0 .
$$

Proof of Proposition 3.5. (a) The claims follow easily from definitions, except for

$$
\tilde{\mathrm{g}}_{\mathbf{R i c}}=0 \quad \text { in } \mathcal{D} \times \mathbb{R} .
$$

On the other hand, this is a well-known consequence of the identities (3.22) and (3.25) satisfied by $\widetilde{h}, \widetilde{X}, \widetilde{Y}$ and $\widetilde{A}$, and the definitions (3.30) and (3.31). See, for example, [12, Section 3] for the proof. 
(b) The identities $\partial_{4} Z^{4}=0, \partial_{4} Z^{a}=0,\left(\mathcal{L}_{Z} \widetilde{\mathbf{g}}\right)_{44}=0,\left(\mathcal{L}_{Z} \widetilde{\mathbf{g}}\right)_{a 4}=0$, and $\left(\mathcal{L}_{Z} \widetilde{\mathbf{g}}\right)_{a b}=0$ give

$$
\begin{aligned}
& Z(\widetilde{X})=0, \quad Z\left(\widetilde{X} \widetilde{A}_{a}\right)+\partial_{a} Z^{c} \widetilde{X} \widetilde{A}_{c}+\partial_{a} Z^{4} \widetilde{X}=0, \\
& Z\left(\widetilde{X}^{-1} \widetilde{h}_{a b}+\widetilde{X} \widetilde{A}_{a} \widetilde{A}_{b}\right)+\partial_{a} Z^{c}\left(\widetilde{X}^{-1} \widetilde{h}_{c b}+\widetilde{X} \widetilde{A}_{c} \widetilde{A}_{b}\right)+\partial_{a} Z^{4} \widetilde{X} \widetilde{A}_{b} \\
& +\partial_{b} Z^{c}\left(\widetilde{X}^{-1} \widetilde{h}_{a c}+\widetilde{X} \widetilde{A}_{a} \widetilde{A}_{c}\right)+\partial_{b} Z^{4} \widetilde{X} \widetilde{A}_{a}=0 .
\end{aligned}
$$

Also using (3.31), it follows that

$$
\begin{aligned}
& Z^{\prime}(\widetilde{X})=0, \quad Z^{\prime}\left(\widetilde{A}_{a}\right)+\partial_{a} Z^{c} \widetilde{A}_{c}+\partial_{a} Z^{4}=0, \\
& Z^{\prime}\left(\widetilde{h}_{a b}\right)+\partial_{a} Z^{c} \widetilde{h}_{c b}+\partial_{b} Z^{c} \widetilde{h}_{a c}=0 .
\end{aligned}
$$

Therefore, along $\mathcal{D}$,

$$
Z^{\prime}(\widetilde{X})=0, \quad\left(\mathcal{L}_{Z^{\prime}}, \widetilde{h}\right)_{a b}=0, \quad\left(\mathcal{L}_{Z^{\prime}} \widetilde{A}\right)_{a}=-\partial_{a} Z^{4} .
$$

The last identity in (3.33),$Z^{\prime}(\tilde{Y})=0$, follows from (3.25), rewritten in the form

$$
\widetilde{\nabla}^{m} \tilde{Y}=-\widetilde{X}^{2} \widetilde{\epsilon}^{a b m} \widetilde{\nabla}_{a} \widetilde{A}_{b} .
$$

We can now complete the proof of the theorem.

Proof of Theorem 1.3. We fix a point $p \in\left(U_{0} \cap \mathcal{H}^{-} \cap \overline{\mathbf{E}}\right) \backslash\left(\mathcal{A} \cup S_{0}\right)$; we may assume that

$$
u_{-}(p)=0, \quad \phi_{-}(p)=0, \quad \theta(p) \in(0, \pi), \quad r(p)=m+\sqrt{m^{2}-a^{2}} .
$$

Then we define the surface $\mathcal{N}_{1}$ as in Proposition 3.3. For any smooth functions $\widetilde{X}, \widetilde{Y}: \mathcal{N}_{1} \rightarrow \mathbb{R}$ agreeing with $X, Y$ in $\mathcal{N}_{1} \cap D_{-}$, we construct the corresponding neighborhood $\mathcal{D}$ of $p$ in $J^{+}\left(\mathcal{N}_{1}\right)$ (which we may assume to be diffeomorphic to the unit ball in $\mathbb{R}^{3}$ and sufficiently small relative to $\left.U_{0}\right)$, the smooth Lorentzian metric $\widetilde{h}$ in $\mathcal{D}$, the scalars $\widetilde{X}, \widetilde{Y}: \mathcal{D} \rightarrow \mathbb{R}$, and the 1-form $\widetilde{A}$, verifying (see (3.22) and (3.25) )

$$
\begin{aligned}
& { }^{\widetilde{h}} \mathbf{R i c}_{a b}=\frac{1}{2 \widetilde{X}^{2}}\left(\nabla_{a} \tilde{X} \nabla_{b} \widetilde{X}+\nabla_{a} \widetilde{Y} \nabla_{b} \tilde{Y}\right), \\
& \widetilde{h} \square(\widetilde{X}+i \widetilde{Y})=\frac{1}{\widetilde{X}} \widetilde{h}^{a b} \partial_{a}(\widetilde{X}+i \widetilde{Y}) \partial_{b}(\tilde{X}+i \widetilde{Y}), \\
& \widetilde{X}^{2}\left(\widetilde{\nabla}_{a} \widetilde{A}_{b}-\widetilde{\nabla}_{b} \widetilde{A}_{a}\right)=\widetilde{\epsilon}_{a b c} \widetilde{\nabla}^{c} \widetilde{Y},
\end{aligned}
$$

in $\mathcal{D}$. Then we construct the space-time metric $\widetilde{\mathbf{g}}$ in $\mathcal{D} \times I$ as in (3.30)-3.31).

In view of Proposition 3.5(a), it remains to show that we can arrange our construction in such a way that the vector-field $Z$ cannot be extended as a Killing vector-field for the modified metric $\widetilde{\mathbf{g}}$. We show first that if $Z$ can be extended as a Killing vector-field for $\widetilde{\mathbf{g}}$, then

$$
\left[Z, \partial_{4}\right]=0 \quad \text { in } \mathcal{D} \times I .
$$

Indeed, letting $V=\left[\partial_{4}, Z\right]$, we calculate, using Lemma 2.2.

$$
\mathbf{D}_{\alpha} \mathbf{D}_{\beta} V_{\mu}=\mathbf{D}_{\alpha} \mathbf{D}_{\beta}\left(\mathcal{L}_{\partial_{4}} Z\right)_{\mu}=V^{\rho} \mathbf{R}_{\rho \alpha \beta \mu} .
$$

Since $V$ vanishes in $\left(\mathcal{D} \cap D_{-}\right) \times I$, it follows that $V$ vanishes in $\mathcal{D} \times I$ as desired. 
The identity 3.35) allows us to apply Proposition 3.5(b). It suffices to prove that we can arrange the construction in subsection 3.2 such that the vector-field $\partial_{3}$ cannot be extended to a vector-field $Z^{\prime}$ in $\mathcal{D}$ such that

$$
\mathcal{L}_{Z^{\prime}} \widetilde{h}=0 \text { and } Z^{\prime}(\widetilde{X})=Z^{\prime}(\widetilde{Y})=0 \text { in } \mathcal{D} .
$$

More precisely, we assume that (3.36) holds and show that there is a choice of $\tilde{X}, \tilde{Y}$ along $\mathcal{N}_{1}$ such that (3.34) is violated.

Assuming that (3.36) holds, we define the geodesic vector-field $\widetilde{L}$ in $\mathcal{D}$ as in subsection 3.2 and notice that

$$
\widetilde{\nabla} \widetilde{L}\left(\widetilde{L}, Z^{\prime}\right)=0
$$

Recall that (see (3.21))

$$
\widetilde{h}(\widetilde{L}, \widetilde{L})=0, \quad\left[\widetilde{L}, Z^{\prime}\right]=0, \quad \widetilde{h}\left(\widetilde{L}, Z^{\prime}\right)=-1, \quad \text { along } \mathcal{N}_{0} .
$$

Since $\widetilde{h}\left(\widetilde{L}, Z^{\prime}\right)=-1$ along $\mathcal{N}_{0}$, it follows that

$$
\widetilde{h}\left(\widetilde{L}, Z^{\prime}\right)=-1 \quad \text { in } \mathcal{D} .
$$

We let $e_{(2)}:=\widetilde{L}, e_{(3)}:=Z^{\prime}$, and fix an additional smooth vector-field $e_{(1)}$ in $\mathcal{D}$ such that $\widetilde{h}\left(e_{(1)}, e_{(2)}\right)=\widetilde{h}\left(e_{(1)}, e_{(3)}\right)=\widetilde{h}\left(e_{(1)}, e_{(1)}\right)-1=0$, i.e.,

$$
e_{(1)}^{a}=\widetilde{\epsilon}^{a b c} \widetilde{L}_{b} Z_{c}^{\prime}
$$

To summarize, assuming (3.36), we have constructed a frame $e_{(1)}, e_{(2)}, e_{(3)}$ in $\mathcal{D}$ such that

$$
\begin{aligned}
\widetilde{h}\left(e_{(1)}, e_{(1)}\right)-1 & =\widetilde{h}\left(e_{(1)}, e_{(2)}\right)=\widetilde{h}\left(e_{(1)}, e_{(3)}\right) \\
& =\widetilde{h}\left(e_{(2)}, e_{(2)}\right)=\widetilde{h}\left(e_{(2)}, e_{(3)}\right)+1=0 .
\end{aligned}
$$

We define the connection coefficients

$$
\Gamma_{(a)(b)(c)}=\widetilde{h}\left(e_{(a)}, \widetilde{\nabla}_{e_{(c)}} e_{(b)}\right) .
$$

Using the identities $\widetilde{\nabla}_{\widetilde{L}} \widetilde{L}=0$ and $\mathcal{L}_{Z^{\prime}} \widetilde{h}=0$, it follows that

$$
\begin{aligned}
& \Gamma_{(a)(2)(2)}=0 \text { for any } a \in\{1,2,3\}, \\
& \Gamma_{(a)(3)(c)}+\Gamma_{(c)(3)(a)}=0 \text { for any }(a, c) \in\{1,2,3\}^{2} .
\end{aligned}
$$

Since $\left[\widetilde{L}, Z^{\prime}\right]=0$ along $\mathcal{N}_{0}$ and

$$
0=\mathcal{L}_{Z^{\prime}}\left(\widetilde{L}^{a} \widetilde{\nabla}_{a} \widetilde{L}_{b}\right)=\widetilde{L}^{a} \widetilde{\nabla}_{a}\left(\mathcal{L}_{Z^{\prime}} \widetilde{L}_{b}\right)+\widetilde{h}^{a c} \widetilde{\nabla}_{a} \widetilde{L}_{b}\left(\mathcal{L}_{Z^{\prime}} \widetilde{L}_{c}\right),
$$

it follows that $\mathcal{L}_{Z^{\prime}} \widetilde{L}=0$ in $\mathcal{D}$. Then, using the definition of $e_{(1)}$, it follows that $\mathcal{L}_{Z^{\prime}} e_{(1)}=0$ in $\mathcal{D}$, therefore

$$
\Gamma_{(a)(3)(c)}=\Gamma_{(a)(c)(3)} \text { for any }(a, c) \in\{1,2,3\}^{2} .
$$

To summarize, letting $F=\widetilde{h}\left(Z^{\prime}, Z^{\prime}\right)=\widetilde{h}_{(3)(3)}$, we have

$$
\begin{array}{ll}
\widetilde{h}_{(1)(1)}-1=\widetilde{h}_{(1)(2)}=\widetilde{h}_{(1)(3)}=\widetilde{h}_{(2)(2)}=\widetilde{h}_{(2)(3)}+1=0, & \widetilde{h}_{(3)(3)}=F, \\
\widetilde{h}^{(1)(1)}-1=\widetilde{h}^{(1)(2)}=\widetilde{h}^{(1)(3)}=\widetilde{h}^{(3)(3)}=\widetilde{h}^{(2)(3)}+1=0, & \widetilde{h}^{(2)(2)}=-F,
\end{array}
$$


and

$$
\begin{aligned}
& \Gamma_{(1)(1)(1)}=\Gamma_{(2)(2)(2)}=\Gamma_{(3)(3)(3)}=\Gamma_{(1)(1)(2)}=\Gamma_{(1)(2)(2)}=\Gamma_{(2)(1)(2)} \\
& \quad=\Gamma_{(2)(2)(1)}=0, \\
& \Gamma_{(3)(2)(2)}=\Gamma_{(2)(3)(2)}=\Gamma_{(2)(2)(3)}=\Gamma_{(3)(1)(1)}=\Gamma_{(1)(3)(1)}=\Gamma_{(1)(1)(3)}=0, \\
& -\Gamma_{(1)(2)(1)}=\Gamma_{(2)(1)(1)}, \\
& -\Gamma_{(3)(3)(a)}=-\Gamma_{(3)(a)(3)}=\Gamma_{(a)(3)(3)}=-\frac{1}{2} e_{(a)}(F), \quad a \in\{1,2\}, \\
& -\Gamma_{(3)(1)(2)}=\Gamma_{(1)(3)(2)}=-\Gamma_{(2)(3)(1)}=\Gamma_{(3)(2)(1)}=-\Gamma_{(2)(1)(3)}=\Gamma_{(1)(2)(3)}, \\
& e_{(3)}(F)=e_{(3)}\left(\Gamma_{(a)(b)(c)}\right)=0, \quad\left[e_{(3)}, e_{(a)}\right]=0, \quad a, b, c \in\{1,2,3\} .
\end{aligned}
$$

We derive now several identities for the connection coefficients $\Gamma$ and the curvature ${ }^{\widetilde{h}} \mathbf{R}$. Clearly,

$$
\begin{aligned}
& { }^{{ }_{h}} \mathbf{R}_{(a)(b)(c)(d)}=\widetilde{h}\left(e_{(a)},\left[\widetilde{\nabla}_{e_{(c)}}\left(\widetilde{\nabla}_{e_{(d)}} e_{(b)}\right)-\widetilde{\nabla}_{e_{(d)}}\left(\widetilde{\nabla}_{e_{(c)}} e_{(b)}\right)-\widetilde{\nabla}_{\left[e_{(c)}, e_{(d)}\right]} e_{(b)}\right]\right) \\
& =\widetilde{h}\left(e_{(a)},\left[\widetilde{\nabla}_{e_{(c)}}\left(\Gamma^{(m)}{ }_{(b)(d)} e_{(m)}\right)-\widetilde{\nabla}_{e_{(d)}}\left(\Gamma^{(m)}{ }_{(b)(c)} e_{(m)}\right)\right.\right. \\
& \left.\left.\quad-\left(\Gamma^{(m)}{ }_{(d)(c)}-\Gamma^{(m)}{ }_{(c)(d)}\right) \widetilde{\nabla}_{e_{(m)}} e_{(b)}\right]\right) \\
& =e_{(c)}\left(\Gamma_{(a)(b)(d)}\right)-e_{(d)}\left(\Gamma_{(a)(b)(c)}\right) \\
& +\Gamma_{(b)(d)}^{(m)} \Gamma_{(a)(m)(c)}-\Gamma_{(b)(c)}^{(m)} \Gamma_{(a)(m)(d)}+\left(\Gamma^{(m)}{ }_{(c)(d)}-\Gamma_{(d)(c)}^{(m)}\right) \Gamma_{(a)(b)(m)}
\end{aligned}
$$

for any $a \in\{1,2\}$ and $b, c, d \in\{1,2,3\}$. Also using the identities (3.38) and (3.39), it follows that

$$
\begin{aligned}
{ }^{\widetilde{h}} \mathbf{R}_{(a)(3)(2)(3)}= & e_{(2)}\left(\Gamma_{(a)(3)(3)}\right)-\Gamma_{(2)(3)(3)} \Gamma_{(a)(3)(2)} \\
& -\Gamma_{(1)(3)(2)} \Gamma_{(a)(1)(3)}+\Gamma_{(3)(3)(2)} \Gamma_{(a)(2)(3)}, \\
{ }^{\widetilde{h}} \mathbf{R}_{(1)(2)(2)(3)}= & e_{(2)}\left(\Gamma_{(1)(2)(3)}\right), \\
{ }^{\widetilde{h}} \mathbf{R}_{(2)(1)(2)(1)}= & e_{(2)}\left(\Gamma_{(2)(1)(1)}\right)+\Gamma_{(2)(1)(1)} \Gamma_{(1)(2)(1)} .
\end{aligned}
$$

We can now obtain our desired contradiction by constructing a pair of smooth functions $\tilde{X}, \tilde{Y}$ along $\mathcal{N}_{1}$ such that not all the identities above (starting with (3.34)) can be simultaneously verified along $\mathcal{N}_{1}$. For this we fix a smooth system of coordinates $y=\left(y^{1}, y^{2}, y^{3}\right)$ in a neighborhood of the point $p$ in $\Pi$ such that

$$
\mathcal{N}_{1}=\left\{q: y^{3}(q)=0\right\}, \quad \mathcal{N}_{0}=\left\{q: y^{2}(q)=0\right\}, \quad L=\widetilde{L}=\frac{d}{d y^{2}} \text { along } \mathcal{N}_{1} .
$$

More precisely, we fix the $L$, as in the unperturbed Kerr, in a neighborhood of $p$ and define first $y^{2}$ such that $y^{2}$ vanishes on $\mathcal{N}_{0}$ and $L\left(y^{2}\right)=1$. Then we complete the coordinate system on $\mathcal{N}_{0}$ and extend it by solving $L\left(y^{1}\right)=L\left(y^{3}\right)=0$.

Assume $\psi: \mathbb{R}^{3} \rightarrow[0,1]$ is a smooth function equal to 1 in the unit ball and vanishing outside the ball of radius 2 . We are looking for functions $\tilde{X}, \tilde{Y}$ of the form

$$
\widetilde{X}(q)=X(q), \quad \widetilde{Y}(q)=Y(q)+\epsilon \psi\left(\left(y(q)-y\left(p^{\prime}\right)\right) / \epsilon\right), \quad q \in \mathcal{N}_{1},
$$

where $p^{\prime}$ is a fixed point in $\mathcal{N}_{1} \cap D_{+}$sufficiently close to $p$, and $(X, Y)$ are as in (3.13). We show below that such a choice leads to a contradiction, for $\epsilon$ sufficiently small. 
Let

$$
V_{1}=\frac{d}{d y^{1}}, \quad V_{2}=\frac{d}{d y^{2}}, \quad V_{3}=\frac{d}{d y^{3}}, \quad e_{(a)}=K_{(a)}^{i} V_{i}
$$

In view of the definitions,

$$
K_{(2)}^{1}=K_{(2)}^{3}=K_{(1)}^{3}=K_{(2)}^{2}-1=0 \quad \text { along } \mathcal{N}_{1} .
$$

We now use the last identity in (3.40) and the first identity in (3.34), along $\mathcal{N}_{1}$. Since ${ }^{\widetilde{h}} \mathbf{R}_{(2)(1)(2)(1)}={ }^{\widetilde{h}} \mathbf{R i c}_{(2)(2)}$, and recalling (3.38) and (3.39), we derive

$$
V_{2}\left(\Gamma_{(2)(1)(1)}\right)-\left(\Gamma_{(2)(1)(1)}\right)^{2}=\frac{1}{2 \widetilde{X}^{2}}\left[V_{2}(\widetilde{X})^{2}+V_{2}(\widetilde{Y})^{2}\right]
$$

along $\mathcal{N}_{1}$. In addition, since

$$
\left[e_{(2)}, e_{(1)}\right]=\left[V_{2}, K_{(1)}^{1} V_{1}+K_{(1)}^{2} V_{2}\right]=V_{2}\left(K_{(1)}^{1}\right) V_{1}+V_{2}\left(K_{(1)}^{2}\right) V_{2}
$$

along $\mathcal{N}_{1}$, it follows that

$$
V_{2}\left(K_{(1)}^{1}\right)=K_{(1)}^{1} \cdot \widetilde{h}\left(\left[e_{(2)}, e_{(1)}\right], e_{(1)}\right)=K_{(1)}^{1} \Gamma_{(2)(1)(1)},
$$

along $\mathcal{N}_{1}$. Using the ansatz (3.41) together with (3.42), and (3.44), it follows that

$$
|G|+\left|V_{2}(G)\right| \lesssim 1 \text { for any } G \in\left\{\Gamma_{(2)(1)(1)}, K_{(1)}^{1}, 1 / K_{(1)}^{1}\right\},
$$

along $\mathcal{N}_{1}$, uniformly for all $p^{\prime} \in \mathcal{N}_{1}$ sufficiently close to $p$ and $\epsilon \leq \epsilon\left(p^{\prime}\right)$ sufficiently small.

Next we use the identity on the second line of (3.40) and the Ricci identity in (3.34), along $\mathcal{N}_{1}$. Since ${ }^{\widetilde{h}} \mathbf{R}_{(1)(2)(2)(3)}=-{ }^{\widetilde{h}} \mathbf{R i c}(1)(2)$, and recalling (3.38), (3.39) we infer that

$V_{2}\left(\Gamma_{(1)(2)(3)}\right)=\frac{-1}{2 \widetilde{X}^{2}}\left[V_{2}(\widetilde{X}) \cdot\left(K_{(1)}^{1} V_{1}+K_{(1)}^{2} V_{2}\right)(\widetilde{X})+V_{2}(\widetilde{Y}) \cdot\left(K_{(1)}^{1} V_{1}+K_{(1)}^{2} V_{2}\right)(\tilde{Y})\right]$, along $\mathcal{N}_{1}$. In addition, using again (3.43), it follows that

$$
\begin{aligned}
V_{2}\left(K_{(1)}^{2}\right) & =-\widetilde{h}\left(\left[e_{(2)}, e_{(1)}\right], e_{(3)}\right)+V_{2}\left(K_{(1)}^{1}\right) \widetilde{h}\left(V_{1}, e_{(3)}\right) \\
& =2 \Gamma_{(1)(2)(3)}+K_{(1)}^{2} V_{2}\left(K_{(1)}^{1}\right) / K_{(1)}^{1}
\end{aligned}
$$

along $\mathcal{N}_{1}$. Using once more the ansatz (3.41) together with (3.46), and (3.47) as well the previously established bounds (3.45), it follows that

$$
|G|+\left|V_{2}(G)\right| \lesssim 1 \text { for any } G \in\left\{\Gamma_{(2)(1)(1)}, K_{(1)}^{1}, 1 / K_{(1)}^{1}, \Gamma_{(1)(2)(3)}, K_{(1)}^{2}\right\},
$$

along $\mathcal{N}_{1}$, uniformly for all $p^{\prime} \in \mathcal{N}_{1}$ sufficiently close to $p$ and $\epsilon \leq \epsilon\left(p^{\prime}\right)$ sufficiently small.

Using the Ricci identity in (3.34), the identities $e_{(3)}(\tilde{X})=e_{(3)}(\tilde{Y})=0$, and the bounds (3.48), it follows that

$$
\sum_{a, b \in\{1,2,3\}}\left|{ }^{\widetilde{h}} \mathbf{R i c}_{(a)(b)}\right| \lesssim 1 \quad \text { along } \mathcal{N}_{1} .
$$

Using the first identity in (3.40) with $a=2$, the identity

$$
{ }^{\widetilde{h}} \mathbf{R}_{(2)(3)(2)(3)}={ }^{\widetilde{h}} \mathbf{R i c}_{(2)(3)}+(1 / 2)\left({ }^{\widetilde{h}} \mathbf{R i c}_{(1)(1)}+F^{\widetilde{h}} \mathbf{R i c}_{(2)(2)}\right) \text {, }
$$


and (3.39), it follows that

$$
\begin{aligned}
& V_{2}(F)=-2 \Gamma_{(2)(3)(3)} \\
& V_{2}\left(\Gamma_{(2)(3)(3)}\right)=-\left(\Gamma_{(1)(2)(3)}\right)^{2}+{ }^{\widetilde{h}} \mathbf{R i c}_{(2)(3)}+(1 / 2)\left({ }^{\widetilde{h}} \mathbf{R i c}_{(1)(1)}+F \cdot{ }^{\widetilde{h}} \mathbf{R i c}_{(2)(2)}\right) .
\end{aligned}
$$

Using again (3.48), it follows that

$$
|F|+\left|V_{2}(F)\right|+\left|V_{2}\left(V_{2}(F)\right)\right| \lesssim 1 \quad \text { along } \mathcal{N}_{1},
$$

uniformly for all $p^{\prime} \in \mathcal{N}_{1}$ sufficiently close to $p$ and $\epsilon \leq \epsilon\left(p^{\prime}\right)$ sufficiently small.

We can now derive a contradiction by examining the second equation in (3.34):

$$
\widetilde{h}^{(a)(b)} \widetilde{\nabla}_{(a)} \widetilde{\nabla}_{(b)}(\tilde{Y})=2 \widetilde{X}^{-1} \widetilde{h}^{(a)(b)} e_{(a)}(\widetilde{X}) e_{(b)}(\tilde{Y}) .
$$

Using (3.45) and (3.49), it follows that

$$
\left|e_{(1)}\left(e_{(1)}(\tilde{Y})\right)-F e_{(2)}\left(e_{(2)}(\widetilde{Y})\right)\right| \lesssim 1 \quad \text { along } \mathcal{N}_{1},
$$

uniformly for all $p^{\prime} \in \mathcal{N}_{1}$ sufficiently close to $p$ and $\epsilon \leq \epsilon\left(p^{\prime}\right)$ sufficiently small. This cannot happen, as can easily be seen by first letting $\epsilon \rightarrow 0$ and then $p^{\prime} \rightarrow p$, taking into account that $F$ and $K_{(1)}^{2}$ vanish along $\mathcal{N}_{0} \cap \mathcal{N}_{1}$.

\section{EXTENSION ACROSS NULL HYPERSURFACES}

Assume in this section that $(\mathbf{M}, \mathbf{g})$ is a 4-dimensional Lorentzian manifold satisfying the Einstein vacuum equations $\operatorname{Ric}(\mathbf{g})=0, p \in \mathbf{M}$ is a fixed point along a smooth null hypersurface $\underline{\mathcal{N}} \subseteq \mathbf{M}$ (given by the level hypersurface of a smooth function $\underline{u}: \mathbf{M} \rightarrow \mathbb{R}$ ) with fixed null vector-field $\underline{L}$ at $p$. Assume that $u: \mathbf{M} \rightarrow \mathbb{R}$ is a smooth optical function transversal to $\underline{\mathcal{N}}$, more precisely,

$$
\mathbf{D}^{\alpha} u \mathbf{D}_{\alpha} u=0 \text { in } \mathbf{M}, u(p)=0, \quad\left(\mathbf{D}^{\alpha} \underline{u} \mathbf{D}_{\alpha} u\right)(p)=-1 .
$$

Let $\mathcal{N}$ be the null hypersurface passing through $p$ generated by the zero level set of u, i.e., $\mathcal{N}=\{x \in \mathbf{M}: u(x)=0\}$ and $L=-\mathbf{g}^{\alpha \beta} \partial_{\alpha} u \partial_{\beta}$ its null geodesic generator. Let

$$
O_{-}:=\{x \in \mathbf{M}: \underline{u}(x)<0\}
$$

and assume that $Z$ is a smooth Killing vector-field in $O_{-}$.

4.1. An extendibility criterion. We extend $Z$ to the neighborhood of $p$ as in (2.3), such that

$$
L^{\alpha} L^{\beta}\left(\mathbf{D}_{\alpha} \mathbf{D}_{\beta} Z_{\mu}-Z^{\rho} \mathbf{R}_{\rho \alpha \beta \mu}\right)=0 .
$$

Theorem 4.1. Recall the assumptions at the beginning of the section. In addition, assume that we have, along the null hypersurface $\mathcal{N}$,

$$
\left(\mathcal{L}_{Z} \mathbf{R}\right)(L, X, L, Y)=0
$$

for any vector-fields $X, Y \in T(\mathbf{M})$ tangent to $\mathcal{N}$. Then there is a neighborhood $U$ of $p$ such that $\mathcal{L}_{Z} \mathbf{g}=0$ in $U$.

Remark 4.2. The sufficient condition (4.2) may be replaced by a sufficient condition at the level of the deformation tensor $\pi$, namely

$$
\left(\mathcal{L}_{Z} \mathbf{g}\right)(X, Y)=0 \quad \text { along } \mathcal{N},
$$

for any vector-fields $X, Y \in T(\mathbf{M})$ tangent to $\mathcal{N}$. Both (4.2) and (4.3) lead to the conclusion (4.9), using the identities (4.5)-(4.8). 
Proof of Theorem 4.1. According to the results proved in section 2.1 we introduce the tensors $W, \pi, \omega, B$, and $P$ as in Definition 2.3. Recall that (see Lemma 2.6)

$$
\pi_{\alpha \mu} L^{\mu}=0, \quad \omega_{\alpha \mu} L^{\mu}=0, \quad P_{\alpha \beta \mu} L^{\mu}=0 .
$$

Since $B=(1 / 2)(\pi+\omega)$ we also have $B_{\alpha \mu} L^{\mu}=0$. We fix a function $y: \mathcal{N} \rightarrow \mathbb{R}$ such that $y$ vanishes on $\mathcal{N} \cap \underline{\mathcal{N}}$ and $L(y)=1$ along $\mathcal{N}$. Then we fix a frame $\left(e_{1}, e_{2}, e_{3}, e_{4}\right)$ along $\mathcal{N}$ such that

$$
\begin{aligned}
& e_{1}, e_{2}, e_{4} \text { are tangent to } \mathcal{N}, \quad e_{4}=L, \quad e_{1}(y)=e_{2}(y)=0, \\
& \mathbf{g}\left(e_{1}, e_{2}\right)=\mathbf{g}\left(e_{a}, e_{a}\right)-1=\mathbf{g}\left(e_{4}, e_{3}\right)+1=\mathbf{g}\left(e_{a}, e_{3}\right)=\mathbf{g}\left(e_{3}, e_{3}\right)=0, \quad a \in\{1,2\} .
\end{aligned}
$$

Our main goal is to show that the tensors $W, B, P$ vanish along $\mathcal{N}$. For any tensor $M=M_{\alpha_{1} \ldots \alpha_{k}}$ and any $s \in \mathbb{Z}$ we define $M^{\geq s}$ any component of the tensor $M$ in the basis $\left(e_{1}, e_{2}, e_{3}, e_{4}\right)$ of signature $\geq s$, where the signature of the component $M_{\alpha_{1} \ldots \alpha_{k}}$ is equal to the difference between the number of 4's and the number of 3's in $\left(\alpha_{1}, \ldots, \alpha_{k}\right)$. Thus, for example,

$$
B^{\geq 0} \in\left\{B_{44}, B_{4 a}, B_{a 4}, B_{43}, B_{34}, B_{a b}: a, b \in\{1,2\}\right\} .
$$

Recall our main transport equations (see Lemma 2.7),

$$
\mathbf{D}_{L} B_{\alpha \beta}=L^{\rho} P_{\rho \beta \alpha}-\mathbf{D}_{\alpha} L^{\rho} B_{\rho \beta}
$$

and

$$
\mathbf{D}_{L} P_{\alpha \beta \mu}=L^{\nu} W_{\alpha \beta \mu \nu}+L^{\nu} B_{\mu}{ }^{\rho} \mathbf{R}_{\alpha \beta \rho \nu}-\mathbf{D}_{\mu} L^{\rho} P_{\alpha \beta \rho},
$$

and our main divergence equation (see Lemma 2.4),

$$
\begin{aligned}
\mathbf{D}^{\alpha} W_{\alpha \beta \gamma \delta}= & B^{\mu \nu} \mathbf{D}_{\nu} \mathbf{R}_{\mu \beta \gamma \delta}+\mathbf{g}^{\mu \nu} P_{\mu \rho \nu} \mathbf{R}^{\rho}{ }_{\beta \gamma \delta} \\
& +P_{\beta \nu \mu} \mathbf{R}^{\mu \nu}{ }_{\gamma \delta}+P_{\gamma \nu \mu} \mathbf{R}^{\mu}{ }_{\beta}{ }^{\nu}{ }_{\delta}+P_{\delta \nu \mu} \mathbf{R}^{\mu}{ }_{\beta \gamma}{ }^{\nu} .
\end{aligned}
$$

In view of the definitions we also have

$$
\mathbf{D}_{\alpha} L_{4}=0, \quad \mathbf{D}_{4} L_{\alpha}=0, \quad \alpha \in\{1,2,3,4\} .
$$

We use equations (4.5) and (4.8), together with the assumption $\mathcal{L}_{Z} \mathbf{R}_{4 a b 4}=0$, $a, b \in\{1,2\}$ to write, schematically,

$$
\left(\mathbf{D}_{L} B\right)^{\geq 0}=\mathcal{M}\left(P^{\geq 1}\right)+\mathcal{M}\left(B^{\geq 0}\right), \quad\left(\mathbf{D}_{L} P\right)^{\geq 1}=\mathcal{M}\left(W^{\geq 2}\right)+\mathcal{M}\left(P^{\geq 1}\right)+\mathcal{M}\left(B^{\geq 0}\right) .
$$

Therefore, also using (4.4),

$$
B^{\geq 0}=0, \quad P^{\geq 1}=0, \quad W^{\geq 2}=0 \quad \text { along } \mathcal{N} .
$$

Using (4.9) and the general symmetries of Weyl fields, equation (4.7) with $(\beta \gamma \delta)=(4 a 4), a \in\{1,2\}$, gives, schematically,

$$
\left(\mathbf{D}_{L} W\right)^{\geq 1}=\mathcal{M}\left(B^{\geq-1}\right)+\mathcal{M}\left(P^{\geq 0}\right)+\mathcal{M}\left(W^{\geq 1}\right) .
$$

Using the transport equations (4.5) and (4.6), together with the identities (4.8) and (4.9) we derive, schematically,

$$
\begin{aligned}
& \left(\mathbf{D}_{L} B\right)^{\geq-1}=\mathcal{M}\left(P^{\geq 0}\right)+\mathcal{M}\left(B^{\geq-1}\right), \\
& \left(\mathbf{D}_{L} P\right)^{\geq 0}=\mathcal{M}\left(W^{\geq 1}\right)+\mathcal{M}\left(B^{\geq-1}\right)+\mathcal{M}\left(P^{\geq 0}\right) .
\end{aligned}
$$

Therefore, (4.9) can be upgraded to

$$
B^{\geq-1}=0, \quad P^{\geq 0}=0, \quad W^{\geq 1}=0 \quad \text { along } \mathcal{N} .
$$


We can now continue this procedure. Using (4.10) and the general symmetries of Weyl fields, equation (4.7) with $(\beta \gamma \delta)=(434)$ and $(\beta \gamma \delta)=(412)$ gives, schematically,

$$
\left(\mathbf{D}_{L} W\right)^{\geq 0}=\mathcal{M}\left(B^{\geq-2}\right)+\mathcal{M}\left(P^{\geq-1}\right)+\mathcal{M}\left(W^{\geq 0}\right) .
$$

The transport equations (4.5) and (4.6), together with the identities (4.8) and (4.10) give, schematically,

$$
\begin{aligned}
& \left(\mathbf{D}_{L} B\right)^{\geq-2}=\mathcal{M}\left(P^{\geq-1}\right)+\mathcal{M}\left(B^{\geq-2}\right), \\
& \left(\mathbf{D}_{L} P\right)^{\geq-1}=\mathcal{M}\left(W^{\geq 0}\right)+\mathcal{M}\left(B^{\geq-2}\right)+\mathcal{M}\left(P^{\geq-1}\right) .
\end{aligned}
$$

Therefore, (4.10) can be upgraded to

$$
B=0, \quad P^{\geq-1}=0, \quad W^{\geq 0}=0 \quad \text { along } \mathcal{N} .
$$

Using (4.11) and the general symmetries of Weyl fields, equation (4.7) with $(\beta \gamma \delta)=(4 a 3), a \in\{1,2\}$, gives, schematically,

$$
\left(\mathbf{D}_{L} W\right)^{\geq-1}=\mathcal{M}\left(P^{\geq-2}\right)+\mathcal{M}\left(W^{\geq-1}\right) .
$$

The transport equation (4.6) and the identities (4.8) and (4.11) give, schematically,

$$
\left(\mathbf{D}_{L} P\right)^{\geq-2}=\mathcal{M}\left(W^{\geq-1}\right)+\mathcal{M}\left(P^{\geq-2}\right) .
$$

Therefore, 4.11) can be upgraded to

$$
B=0, \quad P^{\geq-2}=0, \quad W^{\geq-1}=0 \quad \text { along } \mathcal{N} .
$$

Using (4.12), (4.7) and the general symmetries of Weyl fields, it follows that $\mathbf{D}_{3} W_{4343}=0$ and $\mathbf{D}_{3} W_{4312}=0$ along $\mathcal{N}$. Thus, $\mathbf{D}_{3} W_{4 a 3 b}=0$ along $\mathcal{N}, a, b \in$ $\{1,2\}$. Therefore, the divergence equation (4.7) with $(\beta \gamma \delta)=(a 3 b), a, b \in\{1,2\}$, and the transport equation (4.6) give, schematically,

$$
\left(\mathbf{D}_{L} W\right)^{\geq-2}=\mathcal{M}\left(P^{\geq-3}\right)+\mathcal{M}\left(W^{\geq-2}\right), \quad\left(\mathbf{D}_{L} P\right)^{\geq-3}=\mathcal{M}\left(P^{\geq-3}\right) .
$$

Therefore, we have proved that

$$
B=0, \quad P=0, \quad W=0 \quad \text { along } \mathcal{N} .
$$

To prove now that $B, P, W$ vanish in a full neighborhood of the point $p$ we use Proposition 2.10. Lemma 2.11 and the observation that, for $\epsilon_{0}$ sufficiently small, the functions

$$
f_{ \pm}=\left(\underline{u}+\epsilon_{0}\right)\left( \pm u+\epsilon_{0}\right)
$$

are strongly pseudo-convex in a sufficiently small neighborhood of the point $p$. See [1. Appendix A] for more details.

4.2. A non-extendible example. In this subsection we provide examples showing that Killing vector-fields do not extend, in general, across null hypersurfaces in space-times satisfying the Einstein vacuum equations.

Theorem 4.3. With the notation at the beginning of the section, we further assume that $Z(u)=0$ in $O_{-}$and that $Z$ does not vanish identically in a neighborhood of $p$ in $O_{-}$. Then there is a neighborhood $U$ of $p$ diffeomorphic to the open ball $B_{1} \subseteq \mathbb{R}^{4}$ and a smooth Lorentz metric $\mathbf{h}$ in $U$ such that $\mathbf{R i c}(\mathbf{h})=0$ in $U, \mathbf{h}=\mathbf{g}$ in $O_{-}$, but $Z$ does not admit an extension as a smooth Killing vector-field for $\mathbf{h}$ in $U$. 
In other words, the space-time $(\mathbf{M}, \mathbf{g})$ can be modified in a neighborhood $U$ of $p$, on one side of the null hypersurface $\partial O$, in such a way that the resulting space-time is still smooth and satisfies the Einstein vacuum equations, but the symmetry $Z$ fails to extend to $U$.

Proof of Theorem 4.3. We fix a smooth system of coordinates $\Phi^{p}: B_{1} \rightarrow B_{1}(p)$, $\Phi^{p}(0)=p$, where $B_{r}=\left\{x \in \mathbb{R}^{4}:|x|<r\right\}, r>0$, and $B_{1}(p)$ is an open neighborhood of $p$ in $O$. Let $\partial_{1}, \ldots, \partial_{4}$ denote the induced coordinate vector-fields in $B_{1}(p)$ and let $B_{r}(p)=\Phi^{p}\left(B_{r}\right), r \in(0,1]$. For any smooth function $\phi: B \rightarrow \mathbb{C}$, where $B \subseteq B_{1}(p)$ is an open set, and $j=0,1, \ldots$, we define

$$
\left|\partial^{j} \phi(x)\right|=\sum_{\alpha_{1}, \ldots, \alpha_{j}=1}^{4}\left|\partial_{\alpha_{1}} \ldots \partial_{\alpha_{j}} \phi(x)\right|, \quad x \in B .
$$

We assume that

$$
\mathbf{g}_{\alpha \beta}(p)=\operatorname{diag}(-1,1,1,1)
$$

and, for some constant $A \geq 1$,

$$
\sup _{x \in B_{1}(p)} \sum_{j=1}^{6}\left[\left|\partial^{j} u\right|+\left|\partial^{j} \underline{u}\right|+\sum_{\alpha, \beta=1}^{4}\left|\partial^{j} \mathbf{g}_{\alpha \beta}(x)\right|\right] \leq A .
$$

We will construct the neighborhood

$$
U_{p}=\left\{x \in B_{\epsilon_{0}}(p): u(x)>-\epsilon_{0}^{2}\right\}
$$

for some constant $\epsilon_{0}$ sufficiently small (depending only on the constant $A$ in (4.14)). We define first the hypersurface

$$
\mathcal{N}_{0}=\left\{x \in B_{\epsilon_{0}^{1 / 2}}(p): u(x)=-\epsilon_{0}^{2}\right\} .
$$

Recall that $L=-\mathbf{g}^{\alpha \beta} \partial_{\alpha} u \partial_{\beta}$ and notice that $L$ is tangent to $\mathcal{N}_{0}$. We introduce smooth coordinates $\left(y^{1}, y^{2}, y^{4}\right)$ along the hypersurface $\mathcal{N}_{0}$ in such a way that $y^{4}=0$ on $\underline{\mathcal{N}} \cap \mathcal{N}_{0}$ and $L=\partial_{4}$, where $\partial_{1}, \partial_{2}, \partial_{4}$ are the induced coordinate vector-fields along $\mathcal{N}_{0}$.

We consider smooth symmetric tensors $h$ along $\mathcal{N}_{0}$, such that it coincides with g on $\mathcal{N}_{0} \cap O_{-}$and, on both sides of $\mathcal{N}_{0}$,

$$
h\left(\partial_{4}, \partial_{\alpha}\right)=0, \quad \text { in } \mathcal{N}_{0}, \quad \alpha \in\{1,2,4\} .
$$

Thus, the only non-vanishing components of $h$ are

$$
h_{a b}=h\left(\partial_{a}, \partial_{b}\right), \quad \text { in } \mathcal{N}_{0}, \quad a, b \in\{1,2\} .
$$

We would like to apply Rendall's theorem [11, Theorem 3] to construct the metric $\mathbf{h}$ in the domain of dependence of $\underline{\mathcal{N}} \cup \mathcal{N}_{0}$, such that $\mathbf{h}=\mathbf{g}$ along $\underline{\mathcal{N}}$ and $\mathbf{g}=h$ along $\mathcal{N}_{0}$. The only restriction is that the symmetric tensor $h$ is arranged such that the resulting metric satisfies the Einstein equation

$$
\mathbf{h}^{\alpha \beta} \mathbf{R}\left(L, \partial_{\alpha}, L, \partial_{\beta}\right)=0 \quad \text { along } \mathcal{N}_{0},
$$

with $\mathbf{R}$ the Riemann curvature tensor of $\mathbf{h}$. Recalling the definition of $\mathbf{R}$ and noting that for a space-time metric $\mathbf{h}$ which coincides with $h$ on $\mathcal{N}_{0}$ we must have 
$\mathbf{h}^{3 a}=\mathbf{h}^{33}=0$ and $\mathbf{h}^{a b}=h^{a b}$, (i.e., $h^{a c} h_{c b}=\delta_{a b}$ ), we deduce

$$
\begin{gathered}
\mathbf{h}^{\alpha \beta} \mathbf{R}\left(L, \partial_{\alpha}, L, \partial_{\beta}\right)=-I+I I, \\
I=\sum_{a, b \in\{1,2\}} h^{a b} \mathbf{h}\left(\mathbf{D}_{\partial_{a}} \mathbf{D}_{\partial_{4}}\left(\partial_{4}\right), \partial_{b}\right), \\
I I=\sum_{a, b \in\{1,2\}} h^{a b} \mathbf{h}\left(\mathbf{D}_{\partial_{4}}\left(\mathbf{D}_{\partial_{a}} \partial_{4}\right), \partial_{b}\right) .
\end{gathered}
$$

Thus, imposing the auxiliary condition 7 ,

$$
I \equiv 0 \quad \text { along } \mathcal{N}_{0},
$$

equation (4.16) is equivalent to

$$
\sum_{a, b \in\{1,2\}} h^{a b} h\left(\mathbf{D}_{\partial_{4}}\left(\mathbf{D}_{\partial_{a}} \partial_{4}\right), \partial_{b}\right)=0,
$$

which can be viewed as a constraint equation for the metric $h$ on $\mathcal{N}_{0}$. Indeed, we can introduce a covariant differentiation 8 along $\mathcal{N}_{0}$ compatible with $h$ by the formula

$$
h\left(\nabla_{X} Y, Z\right)=\frac{1}{2}[-Z h(X, Y)+Y h(X, Z)+X h(Y, Z)]
$$

for $X, Y, Z \in\left\{\partial_{1} . \partial_{2}, \partial_{4}\right\}$. With this definition we observe that (4.19) is equivalent to

$$
\sum_{a, b \in\{1,2\}} h^{a b} h\left(\nabla_{\partial_{4}}\left(\nabla_{\partial_{a}} \partial_{4}\right), \partial_{b}\right)=0 .
$$

In view of the definition (4.20), for $a \in\{1,2\}$,

$$
\begin{aligned}
& \nabla_{\partial_{a}} \partial_{4}=(1 / 2) h^{c d}\left(\partial_{4} h_{a d}\right) \partial_{c}+\operatorname{multiple}\left(\partial_{4}\right), \\
& \nabla_{\partial_{4}} \partial_{a}=(1 / 2) h^{c d}\left(\partial_{4} h_{a d}\right) \partial_{c}+\operatorname{multiple}\left(\partial_{4}\right) .
\end{aligned}
$$

Therefore, the identity (4.21) is equivalent to

$$
\partial_{4}\left(h^{a d} \partial_{4} h_{a d}\right)+(1 / 2) h^{a b} h^{c d} \partial_{4} h_{a d} \partial_{4} h_{b c}=0 .
$$

Letting

$$
h_{a b}=\phi^{2} \widehat{h}_{a b}, \quad \operatorname{det}(\widehat{h})=\widehat{h}_{11} \widehat{h}_{22}-\widehat{h}_{12}^{2}=1,
$$

and making the observation $\widehat{h}^{a d} \widetilde{\partial}_{4} \widehat{h}_{a d}=0$, the identity (4.22) is equivalent to

$$
\partial_{4}^{2} \phi+(1 / 8) \phi \cdot \widehat{h}^{a b} \widehat{h}^{c d} \partial_{4} \widehat{h}_{a d} \partial_{4} \widehat{h}_{b c}=0 .
$$

In other words, we may define $\widehat{h}_{a b}, a, b \in\{1,2\}$, as an arbitrary smooth positive definite symmetric tensor along $\mathcal{N}_{0}$, with $\widehat{h}_{11} \widehat{h}_{22}-\widehat{h}_{12}^{2}=1$ and $\widehat{h}_{a b}=\left(\mathbf{g}_{11} \mathbf{g}_{22}-\right.$ $\left.\mathbf{g}_{12}^{2}\right)^{-1 / 2} \mathbf{g}\left(\partial_{a}, \partial_{b}\right)$ in $\mathcal{N}_{0} \cap O_{-}$. We then define $\phi$ according to equation (4.23), and the full tensor $h=\phi^{2} \widehat{h}$ along $\mathcal{N}_{0}$. Finally, we apply Rendall's theorem 11, Theorem 3] (or Theorem 2 in [9]) to construct a smooth space-time metric $\mathbf{h}$ in $\widetilde{U}_{p}=\{x \in$ $\left.B_{\epsilon_{0}}(p): u(x) \geq-\epsilon_{0}^{2}\right\}$ satisfying the Einstein vacuum equations and agreeing with g in $U_{p} \cap O_{-}$and with $h$ along $\mathcal{N}_{0} \cap B_{\epsilon_{0}}$.

${ }^{7}$ Writing $\mathbf{D}_{\partial_{4}} \partial_{4}=\omega \partial_{4}$, the condition reduces to $\omega=0$ along $\mathcal{N}_{0}$.

${ }^{8}$ Since the metric $h$ is degenerate on $\mathcal{N}_{0}$, this formula only defines the covariant derivatives $\nabla_{X} Y$ up to a multiple of $L=e_{4}$. 
It remains to prove that we can arrange $\widehat{h}_{a b}$ on $\mathcal{N}_{0}$ such that $Z$ does not admit an extension to $U_{p}$ as a Killing vector-field for $\mathbf{h}$. We extend the smooth vector-field $L$ from $U_{p} \cap O_{-}$to all of $U_{p}$ such that

$$
\mathbf{D}_{L} L=0 \text { in } U_{p}
$$

with $\mathbf{D}$ the covariant differentiation associated to the metric $\mathbf{h}$.

Since $Z(u)=0$ in $O_{-}$it follows that $[Z, L]=0$ in $O_{-}$. Assume, for contradiction, that $Z$ admits an extension to $U_{p}$ as a Killing vector-field for $\mathbf{h}$. Then, letting $V=\mathcal{L}_{Z} L$, we compute in $U_{p}$

$$
L^{\rho} \mathbf{D}_{\rho} V_{\alpha}=L^{\rho} \mathcal{L}_{Z} \mathbf{D}_{\rho} L_{\alpha}=-V^{\rho} D_{\rho} L_{\alpha} .
$$

Since $V$ vanishes in $U_{p} \cap O_{-}$, it must vanish in all of $U_{p}$, i.e.,

$$
[L, Z]=0 \quad \text { in } U_{p} \text {. }
$$

In addition, since

$$
L \mathbf{h}(Z, L)=0
$$

we infer that $Z$ must remain tangent to the hypersurface $\mathcal{N}_{-\epsilon_{0}^{2}}$. To summarize, by contradiction, we have constructed a vector-field $Z$ in $U_{p}$ tangent to the hypersurface $\mathcal{N}_{0}$ such that, on $\mathcal{N}_{0} \cap U_{p}$,

$$
\mathcal{L}_{Z} h=0, \quad[L, Z]=0 .
$$

On the other hand, writing $Z=Z^{1} \partial_{1}+Z^{2} \partial_{2}+Z^{4} \partial_{4}$ in the system of coordinates along $\mathcal{N}_{0}$ introduced before, the identity $\mathcal{L}_{Z} h=0$ in (4.24) gives

$$
0=Z\left(h_{a b}\right)+\partial_{a} Z^{\rho} h_{\rho b}+\partial_{b} Z^{\rho} h_{a \rho}, \quad a, b \in\{1,2\} .
$$

Therefore,

$$
Z(\operatorname{det}(h))=Z\left(h_{11} h_{22}-h_{12}^{2}\right)=-2\left(\partial_{1} Z^{1}+\partial_{2} Z^{2}\right) \operatorname{det}(h) .
$$

Since $h=(\operatorname{det} h)^{1 / 2} \widehat{h}$, the identity $\mathcal{L}_{Z} h=0$ shows that

$$
\mathcal{L}_{Z} \widehat{h}=\left(\partial_{1} Z^{1}+\partial_{2} Z^{2}\right) \widehat{h} .
$$

Notice also that $Z$ does not depend on the choice of the tensor $\widehat{h}$; indeed, $Z$ is defined simply by the relation $[L, Z]=0$ in (4.24). Therefore, we obtain a contradiction by choosing $\widehat{h}$ such that (4.25) fails at some point in $\mathcal{N}_{0} \backslash O_{-}$. This completes the proof.

Remark. We can interpret condition (4.18) using the null second fundamental form of $\mathcal{N}_{0}$ with respect to the $\mathbf{h}$ metric,

$$
\chi(X, Y):=\mathbf{h}\left(\mathbf{D}_{X} L, Y\right), \quad \forall X, Y \quad \text { tangent to } \mathcal{N}_{0} .
$$

Clearly, $\mathbf{D}_{L} L=\omega L$ along $\mathcal{N}_{0}$ for some smooth function $\omega$. Thus,

$$
\begin{aligned}
I & =\sum_{a, b \in\{1,2\}} h^{a b} \mathbf{h}\left(\mathbf{D}_{\partial_{a}} \mathbf{D}_{\partial_{4}}\left(\partial_{4}\right), \partial_{b}\right) \\
& =\sum_{a, b \in\{1,2\}} \omega h^{a b} \mathbf{h}\left(\mathbf{D}_{\partial_{a}} \partial_{4}, \partial_{b}\right)=\omega h^{a b} \chi_{a b}=\omega \operatorname{tr} \chi .
\end{aligned}
$$

Thus (4.18) takes the form

$$
\omega \cdot \operatorname{tr} \chi=0
$$

from which we infer that $\omega$ must vanish in $U_{p} \cap \mathcal{N}_{0}$ (i.e., $\mathbf{D}_{L} L=0$ ) if $\operatorname{tr} \chi$ vanishes at most on a set with empty interior in $\left(U_{p} \cap \mathcal{N}_{0}\right) \backslash O_{-}$. 
On the other hand,

$$
\chi_{a b}=\frac{1}{2} \partial_{4} h_{a b}=\frac{1}{2} \partial_{4}\left(\phi^{2} \widehat{h}_{a b}\right)=\phi \partial_{4} \phi \widehat{h}_{a b}+\frac{1}{2} \phi^{2} \partial_{4} \widehat{h}_{a b},
$$

from which

$$
\operatorname{tr} \chi=\phi^{-2} \widehat{h}^{a b}\left(\phi \partial_{4} \phi \widehat{h}_{a b}+\frac{1}{2} \phi^{2} \partial_{4} \widehat{h}_{a b}\right)=2 \phi^{-1} \partial_{4} \phi .
$$

Also, the traceless part of $\chi$ satisfies the identity

$$
\hat{\chi}_{a b}=\chi_{a b}-\frac{1}{2} \operatorname{tr} \chi h_{a b}=\frac{1}{2} \phi^{2} \partial_{4} \widehat{h}_{a b} .
$$

Thus, equation (4.23) takes the well-known form

$$
\partial_{4} \operatorname{tr} \chi+\frac{1}{2}(\operatorname{tr} \chi)^{2}=-|\hat{\chi}|_{h}^{2}
$$

from which we infer that $\operatorname{tr} \chi$ can only vanish in a set with empty interior in $\left(U_{p} \cap \mathcal{N}_{0}\right) \backslash O_{-}$if the same holds true for $\hat{\chi}$. Thus, we can easily choose non-trivial data on $\mathcal{N}_{0}$ such that, for our original choice of $L=\partial_{4}$, we have

$$
\mathbf{D}_{L} L=0 \quad \text { in } \mathcal{N}_{0} \cap U_{p} \text {. }
$$

\section{REFERENCES}

[1] Spyros Alexakis, Alexandru D. Ionescu, and Sergiu Klainerman, Hawking's local rigidity theorem without analyticity, Geom. Funct. Anal. 20 (2010), no. 4, 845-869, DOI 10.1007/s00039010-0082-7. MR.2729279 (2012c:53113)

[2] S. Alexakis, A. D. Ionescu, and S. Klainerman, Uniqueness of smooth stationary black holes in vacuum: small perturbations of the Kerr spaces, Comm. Math. Phys. 299 (2010), no. 1, 89-127, DOI 10.1007/s00220-010-1072-1. MR2672799 (2012d:53239)

[3] Demetrios Christodoulou and Sergiu Klainerman, The global nonlinear stability of the Minkowski space, Princeton Mathematical Series, vol. 41, Princeton University Press, Princeton, NJ, 1993. MR 1316662 (95k:83006)

[4] Piotr T. Chruściel, On rigidity of analytic black holes, Comm. Math. Phys. 189 (1997), no. 1, 1-7, DOI 10.1007/s002200050187. MR 1478527 (98j:83054)

[5] S. W. Hawking and G. F. R. Ellis, The large scale structure of space-time, Cambridge University Press, London, 1973. Cambridge Monographs on Mathematical Physics, No. 1. MR0424186 (54 \#12154)

[6] Lars Hörmander, The analysis of linear partial differential operators. IV, Grundlehren der Mathematischen Wissenschaften [Fundamental Principles of Mathematical Sciences], vol. 275, Springer-Verlag, Berlin, 1985. Fourier integral operators. MR781537 (87d:35002b)

[7] Alexandru D. Ionescu and Sergiu Klainerman, On the uniqueness of smooth, stationary black holes in vacuum, Invent. Math. 175 (2009), no. 1, 35-102, DOI 10.1007/s00222-008-0146-6. MR2461426 (2009j:83053)

[8] Alexandru D. Ionescu and Sergiu Klainerman, Uniqueness results for ill-posed characteristic problems in curved space-times, Comm. Math. Phys. 285 (2009), no. 3, 873-900, DOI 10.1007/s00220-008-0650-y. MR2470908 (2010j:83075)

[9] J. Luk, On the local existence for the characteristic initial value problem in general relativity, Preprint (2011).

[10] Katsumi Nomizu, On local and global existence of Killing vector fields, Ann. of Math. (2) 72 (1960), 105-120. MR0119172 (22 \#9938)

[11] A. D. Rendall, Reduction of the characteristic initial value problem to the Cauchy problem and its applications to the Einstein equations, Proc. Roy. Soc. London Ser. A 427 (1990), no. 1872, 221-239. MR1032984 (91a:83004)

[12] Gilbert Weinstein, On rotating black holes in equilibrium in general relativity, Comm. Pure Appl. Math. 43 (1990), no. 7, 903-948, DOI 10.1002/cpa.3160430705. MR1072397 (91h:83063) 
LOCAL EXTENSION OF KILLING VECTOR-FIELDS IN RICCI FLAT MANIFOLDS 593

Department of Mathematics, Princeton University, Princeton, New Jersey 08544

E-mail address: aionescu@math.princeton.edu

Department of Mathematics, Princeton University, Princeton, New Jersey 08544

E-mail address: seri@math.princeton.edu 